OPEN ACCESS

Edited by:

Zhen Cheng,

Stanford University, United States

Reviewed by:

Zhenhua Hu,

University of Chinese Academy of

Sciences, China

Adam Glaser

University of Washington,

United States

*Correspondence:

Petr Bruza

Petr.Bruza@dartmouth.edu

†These authors have contributed equally to this work

Specialty section

This article was submitted to Medical Physics and Imaging,

a section of the journa Frontiers in Physics

Received: 05 June 2020

Accepted: 15 July 2020

Published: 21 August 2020

Citation:

Ashraf MR, Rahman M, Zhang R,

Williams BB, Gladstone DJ,

Pogue BW and Bruza P (2020) Dosimetry for FLASH Radiotherapy: A

Review of Tools and the Role of

Radioluminescence and Cherenkov

Emission. Front. Phys. 8:328

doi: 10.3389/fphy.2020.00328

\section{Dosimetry for FLASH Radiotherapy: A Review of Tools and the Role of Radioluminescence and Cherenkov Emission}

\author{
Muhammad Ramish Ashraf ${ }^{1 \dagger}$, Mahbubur Rahman ${ }^{1 \dagger}$, Rongxiao Zhang ${ }^{1,2,3}$, \\ Benjamin B. Williams ${ }^{1,2,3}$, David J. Gladstone ${ }^{1,2,3}$, Brian W. Pogue ${ }^{1,3}$ and Petr Bruza ${ }^{1 *}$ \\ ${ }^{1}$ Thayer School of Engineering, Dartmouth College, Hanover, NH, United States, ${ }^{2}$ Department of Medicine, Geisel School of \\ Medicine, Dartmouth College, Hanover, NH, United States, ${ }^{3}$ Norris Cotton Cancer Center, Dartmouth-Hitchcock Medical \\ Center, Lebanon, NH, United States
}

While spatial dose conformity delivered to a target volume has been pushed to its practical limits with advanced treatment planning and delivery, investigations in novel temporal dose delivery are unfolding new mechanisms. Recent advances in ultra-high dose radiotherapy, abbreviated as FLASH, indicate the potential for reduction in healthy tissue damage while preserving tumor control. FLASH therapy relies on very high dose rate of > 40Gy/s with sub-second temporal beam modulation, taking a seemingly opposite direction from the conventional paradigm of fractionated therapy. FLASH brings unique challenges to dosimetry, beam control, and verification, as well as complexity of radiobiological effective dose through altered tissue response. In this review, we compare the dosimetric methods capable of operating under high dose rate environments. Due to excellent dose-rate independence, superior spatial $(\sim<1 \mathrm{~mm})$ and temporal $(\sim \mathrm{ns})$ resolution achievable with Cherenkov and scintillation-based detectors, we show that luminescent detectors have a key role to play in the development of FLASH, as the field rapidly progresses toward clinical adaptation. Additionally, we show that the unique ability of certain luminescence-based methods to provide tumor oxygenation maps in real-time with submillimeter resolution can elucidate the radiobiological mechanisms behind the FLASH effect. In particular, such techniques will be crucial for understanding the role of oxygen in mediating the FLASH effect.

Keywords: Cherenkov, radioluminescence, optical imaging, FLASH, dosimetry, scintillation

\section{INTRODUCTION}

Decades of research in radiation therapy has been focused toward increasing the therapeutic ratio [1] (Figure 1A), and many techniques such as inverse treatment planning optimization, intensity modulated radiation therapy, or on-board imaging guidance have achieved this goal primarily via higher spatial modulation of the primary beam (Figure 1B). Temporal modulation of dose has also been widely exploited in its relation to repair of sublethal damage and the cell cycle, and this has been widely adopted in almost all clinical treatments through fractionated treatment plans. Yet, the role of higher dose-rate effects has been largely undeveloped in clinical treatment [3]. 
Interesting early studies [4-8] (1960-1980) had observed peculiar effects of reduced cell killing at ultra-high dose-rates, such as the study by Hornsey et al. [2], who illustrated reduced cell killing in mouse intestine at high dose rates (Figure 1C). However, a recent study (2014) by Favaudon et al. [9] has sparked explosive interest in ultra-high dose rates again. Contrary to conventional radiotherapy techniques, which employ mean doserates of $\sim 0.03 \mathrm{~Gy} / \mathrm{s}$ with doses of $\sim 2 \mathrm{~Gy}$ delivered over 1030 fractions, the authors used an ultra-high mean-dose rate of $40 \mathrm{~Gy} / \mathrm{s}$ with total irradiation time $<500 \mathrm{~ms}$ to achieve an improved differential response between tumor and normal tissue. They reported less normal tissue damage with high doserate irradiation when compared to "conventional" radiotherapy conditions, while observing similar anti-tumor response in both modalities. The authors termed this phenomenon as the "FLASH" effect. Multiple groups have now replicated the FLASH effect in different murine organs $[10,11]$ and in superficial treatments in animal models such as mini-pigs, cats [12] and zebrafish [13]. It has been shown that the FLASH effect can be triggered using electrons [9, 14, 15], x-rays [16, 17], and protons $[18,19]$. Recently, the first human patient was treated with FLASH [18], with promising clearance of the lymphoma lesion with lower skin toxicity than has been seen in previous irradiations. The field of FLASH has experienced wide interest and growth and could be a critical area of development for better normal tissue sparing in radiotherapy. The calibration and quality assurance tools for dosimetry also need to be adapted accordingly to keep up with the ever-changing nature of radiation dose delivery Historically, spatial modulation in dose delivery has led to increased use of small radiation fields or beamlets for which accurate dosimetric characterization was found to be non-trivial. These problems associated with small fields are well-documented [20]. This warranted a need for highresolution detectors which most vendors now typically provide for the measurement of cumulative dose distributions [21]. With the emergence of FLASH and other promising high dose-rate modalities, such as Microbeam Radiation Therapy (MRT) [22] and Synchrotron stereotactic radiotherapy [23], it is expected that new dosimetric challenges will arise. Spatiotemporal dosimetry for small radiation beamlets delivered dynamically under high dose-rate conditions can be difficult. For successful translation of FLASH to a clinical setting, dosimetry must be performed accurately and rigorously keeping in mind limitations of various detectors at high dose-rates and non-standard nature of the various FLASH irradiation platforms. The issue of dosimetric uncertainty in preclinical radiobiological studies and its effect on reproducibility and eventual translation to clinics has been highlighted by multiple authors [24, 25]. The National Institute of Standards and Technology (NIST) has recommendations regarding accurate measurement and reporting of dose in preclinical radiobiological studies [26]. In a multi-institution audit of irradiator output [25], it was found that only one facility was able to deliver a dose within $5 \%$ of the prescribed limit; other facilities had errors ranging from 12 to $42 \%$. The issue is primarily because of the non-standard nature of irradiation platforms used in pre-clinical studies and a lack of protocols and guidance. To tackle this issue, the American Association of Physicists in
Medicine (AAPM) has initiated Task Group No. 319 "Guidelines for accurate dosimetry in radiation biology experiments." One of the major aims of the task group is to standardize dosimetry and review uncertainties associated with non-clinical units used in preclinical radiobiological studies. Therefore, this review is motivated by the need to assess the various different uncertainties associated with performing accurate dosimetry under FLASH and high dose-rate irradiation conditions.

To this end, dosimetric problems unique to high dose-rate environment such as FLASH will be identified in this review. Common radiation dosimeters based on different physical principles, mainly, luminescent, charged based and chemical detectors will be discussed in light of the dosimetric issues identified earlier. We hypothesize that based on the underlying physical mechanisms, luminescent based detectors can be used to perform accurate, real-time dosimetry, with predetermined corrections under reference conditions to account for nonideal characteristics (e.g., quenching of scintillator response for high LET particles and energy dependence of Cherenkov radiation). It can be argued that the most important dosimetric aspects of FLASH (discussed in detail in section Dosimetric Aspects of FLASH That Need to be Considered) are dose-rate independence, spatial resolution, and temporal resolution of a detector. Comparing these three characteristics of the detectors, their typical usage, and the underlying physics, it can be predicted that luminescent detectors offer unparalleled spatial-temporal resolution and dose-rate independence (Figure 2). Values in Figure 2 are based on typical usage and exceptions to these do exist; these exceptions will be noted, where appropriate, in the text. Another purpose of this review is to provide an overview of tools that have been used in high-dose rate conditions. Therefore, instances of different dosimetric tools used in FLASH and other high dose-rate modalities will be consolidated and summarized. Finally, the mediating role of oxygen tension in FLASH will be discussed briefly. The superior ability of luminescent based methods to sense oxygen tension and measure dose and LET (simultaneously, specifically for particle therapy) in real-time will be described for its potential in understanding the radiobiological mechanisms underlying the protective effect of FLASH on normal tissue.

\section{DOSIMETRIC ASPECTS OF FLASH THAT NEED TO BE CONSIDERED}

\section{Dose-Rate Dependency}

It is instructive to first compare temporal structure of FLASH and conventional radiotherapy beams in order to understand problems unique to high dose-rate conditions. Typically, radiation sources used in radiotherapy are not continuous but rather pulsatile in nature. The repetition rate and duty cycle depend on the type of particle acceleration used. For instance, most clinical linear accelerators (linac) typically have a pulse duration of 3-5 $\mu$ s with repetition rate of $\sim 200-$ $400 \mathrm{~Hz}$. For cyclotron based proton beams, the beam can be considered to be quasi-continuous due to short pulse duration and repetition rates on the order of a few nanoseconds. 

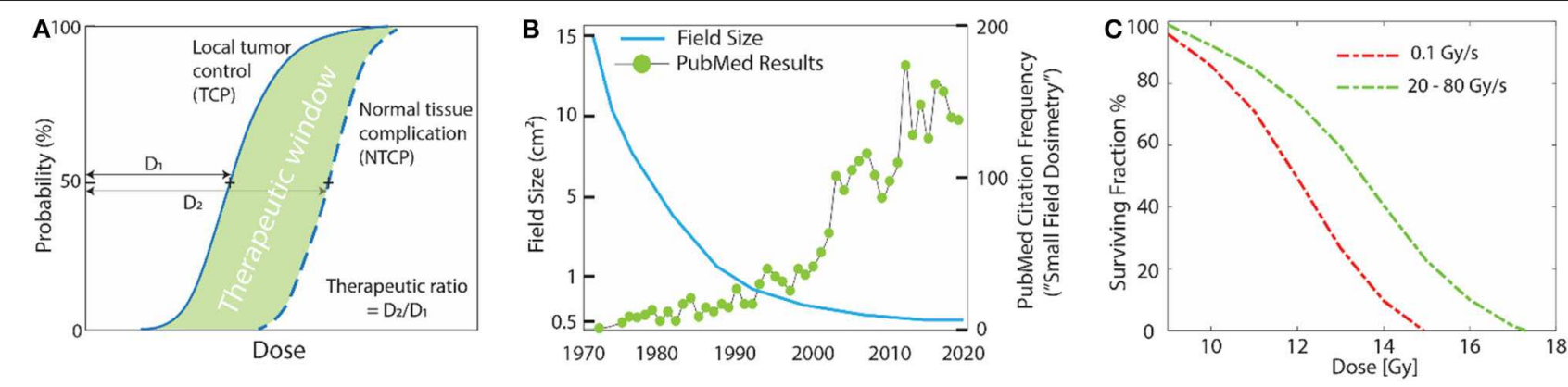

FIGURE 1 | (A) Typical dose-response curves in radiotherapy; maximum tumor control with minimal normal tissue complication is desired. (B) Over last the few decades this has been possible due to spatial modulation of the beam, leading to an increasing use of small fields (<10 mm). Unfortunately, this led to complex dosimetric issues unique to small fields that standard dosimeters were not suitable for. The x-axis in the in (B) denotes the time-line of major advancements that have happened in the field of external beam radiotherapy. Left scale denotes how typical field sizes have varied with these advancements and right scale shows the number of peer-reviewed publications per year based on a PubMed search of the phrase "Small Field Dosimetry." (C) The effect of ultra-high dose rates on cell survival curves. Data adapted from Hornsey et al. [2], where decreased cell killing was seen in mouse intestine at high dose-rates vs. low dose-rates. The decrease in cell-killing was attributed to rapid depletion of oxygen, which is required to "fix" DNA damage, at high dose-rates.

Additionally, modern radiation techniques typically deliver dose in fractionated manner over a period of few days. Therefore, dose-rate can be either the defined over the course of a whole treatment, one fraction or within a single pulse. The different interpretations of dose-rate are illustrated in Figure 3A. Table in Figure 3B presents a side by side comparison of temporal beam characteristics of FLASH and CONV radiation therapy; the dose per fraction is denoted as the mean dose-rate, $\dot{\mathrm{D}}_{\mathrm{m}}$. The dose-rate in a pulse or the instantaneous dose-rate is denoted as $\dot{\mathrm{D}}_{\mathrm{p}}$, which is the ratio of dose delivered in a pulse $\left(D_{p}\right)$ divided by the pulse duration. This is an important distinction because instantaneous dose-rates in conventional radiotherapy can be comparable or even higher when compared to the mean dose-rate $\left(\dot{D}_{m}\right)$ of $40 \mathrm{~Gy} / \mathrm{s}$ used to trigger the FLASH effect in current preclinical studies. Note that the conventional beam characteristics are based on typical linac based clinical beams and the values for FLASH are based on prototype electron linacs that have been successfully used to elicit the FLASH effect [27]. Multiple studies have now rigorously explored the beam parameters needed to trigger a reproducible FLASH effect and it has been shown that the $\dot{\mathrm{D}}_{\mathrm{p}}$ and $\mathrm{D}_{\mathrm{p}}$ play a critical role $[27,28]$. For FLASH, these quantities can be orders of magnitude higher as shown in $3 \mathrm{~b}$, leading to issues of saturation, and non-linear response of standard dosimeters at large doses.

\section{Spatial Resolution}

Many preclinical FLASH studies have been performed on small animal models and tumor volumes. As mentioned earlier, performing accurate dosimetry with small beamlets is notoriously difficult. If the size of the sensitive volume of the detector is comparable to the radiation field size, dose averaging effects can lead to erroneous measurement of absorbed dose and artificial broadening of the field penumbra. Multiple preclinical studies have been performed using the experimental Oriatron eRT6- 6MeV and Kinetron 4.5 MeV linear accelerator (PMB-Alcen, Peynier, France). Design limitations of these linacs result in only small field openings. Small applicators used

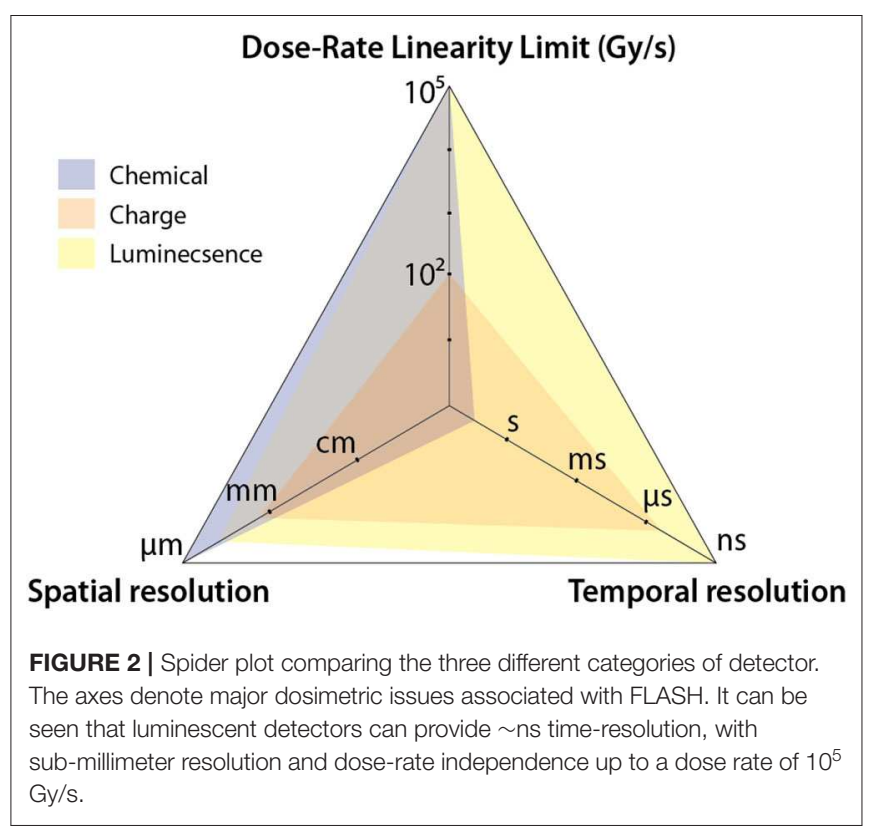

to confine the radiation field to the organs of interest can also introduce additional complex problems [29]. Additionally, clinical linacs modified to deliver flash dose rates [30] tends to utilize significantly shorter source to target distance to further increase the dose rate. Close proximity to source renders only small field sizes viable. Varian's Clinac 21EX was modified by Schüler et al. [30] and it was found that mean dose-rate of 900 Gy/s was achievable near the transmission ionization chamber in the gantry head, where $90 \%$ of the field diameter was measured to be $\sim 12 \mathrm{~mm}$. For the beam energy used in the study $(20 \mathrm{MeV})$, this falls in the realm of small field dosimetry, due to the lateral electronic disequilibrium. In general, a detector with spatial resolution of $<1 \mathrm{~mm}$ is desired. However, it should be noted that different prospective FLASH radiotherapy sources with radically 


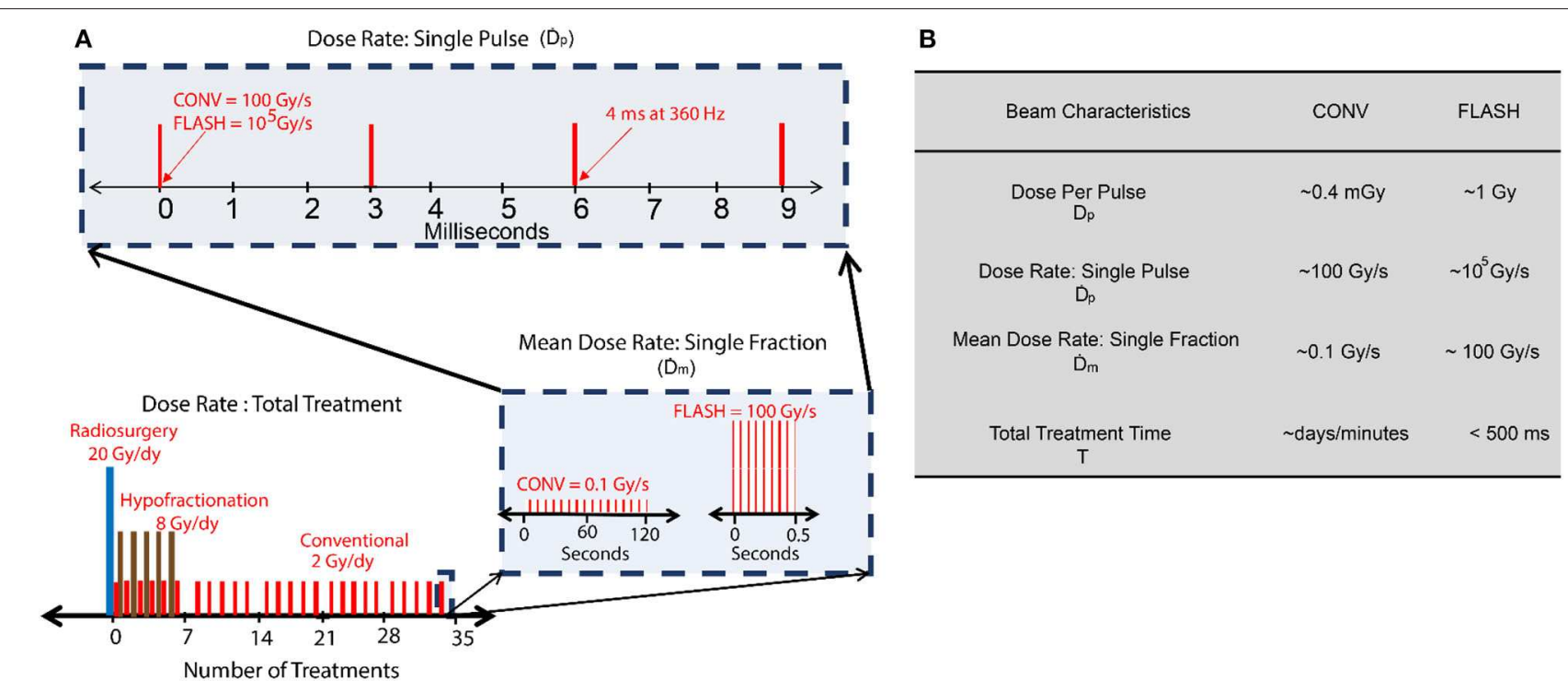

FIGURE 3 | (A) Dose rate schemes in radiation therapy indicating different interpretations of dose-rate. (B) Typical temporal beam characteristics for conventional (CONV) and FLASH using electrons.

different spatial requirements are currently being investigated [27]. Conventional electron linear accelerators typically operate in the 1-25 MeV range and thus offer poor depth penetration. Hence, there is considerable interest shown in other radiotherapy sources such as Very High Energy Electron (VHEE) beams, laser plasma accelerators, synchrotron and cyclotron based sources $[31,32]$. In the context of small field dosimetry, these prospective FLASH source brings along complex challenges, especially those operating with coherent radiation beams. Microbeam Radiation Therapy (MRT), and other forms of highly spatially fractionated radiation therapy, typically deliver dose at high dose-rates with field sizes on the order of few micrometers. In this case, a spatial resolution of $\sim 1 \mathrm{~mm}$ will be inadequate. As MRT has been shown to trigger the FLASH effect in vivo [33], a micrometer resolution is needed for some of the more exotic FLASH modalities.

Another issue specific to FLASH is that of spatial averaging of dose-rate. Accurate determination of fully spatially-resolved dose-rate is critical for FLASH accuracy. This is even more important currently, since one of the main goals is to optimize the beam parameters so that a FLASH effect can be elicited in the clinical setting. A simulation study conducted by Marlen et al. [34] explored the idea of spatial dose-rate distribution within a broad beam and its effect on FLASH response. Their study was based on pencil beam scanning with protons, but the results can be generalized to other modalities. Large field sizes are obtainable with protons when spot scanning techniques are used. However, as reported in the study, dose rate in one spot will be affected by the low dose-rate scatter contributed by the adjacent spots (shown in Figure 4). They reported that only $40 \%$ of the dose is delivered at FLASH rates for a spot peak dose rate at the center of $100 \mathrm{~Gy} / \mathrm{s}$. When the peak dose rate is increased to 360 $\mathrm{Gy} / \mathrm{s}$, the contribution to FLASH dose rates increases to $75 \%$. Rahman et al. also measured, using a scintillating sheet, as much as $41 \%$ standard deviation in maximum dose rate distribution for spot spacing as large as $10 \mathrm{~mm}$ (shown in Figure 10A) [35]. This will also hold true for broad electron beams, synchrotron produced $\mathrm{x}$-ray beams and passively scattered proton beams, where scatter can contribute to an inhomogeneous distribution of dose-rates across the irradiated volume. This phenomena was also studied by Van de Water et al. [36] where they investigated the possibility of achieving FLASH dose-rates with conventional proton pencil beam scanning and intensity modulated proton therapy techniques. They proposed a new metric to quantify spatially varying dose-rate in three dimensions, dose averaged dose-rate (DADR) and dose-weighted average of instantaneous dose-rate of all spots. It remains to be seen if such a criterion has any potential clinical value, but it points toward the need of a high resolution imaging detector. Additionally, as FLASH continues to move toward clinical translation, large field sizes will need to be delivered at high dose-rates. Spatial distribution of dose and dose-rate can only be reliably measured using imaging techniques or detector arrays. Thus, a high resolution detector array with small inter-detector spacing is needed, so that a $2 \mathrm{D}$ spatial distribution of dose-rate can be measured accurately, avoiding any volume averaging effects. A 3D distribution of dose-rate within patient geometry would be the ideal case.

\section{Time Resolution}

Another aspect of dosimetry under FLASH conditions is that of real-time dose monitoring vs. passive dose monitoring. While dose-rate independence is an important requirement for FLASH, the ability to verify machine output, dose delivered per pulse, and dose-rate in real-time is of considerable interest. For high doserates and dose per pulse conditions, real-time dose monitoring is non-trivial. Not only dose-rate independent dosimeters are required, but dosimeters with a high enough temporal 


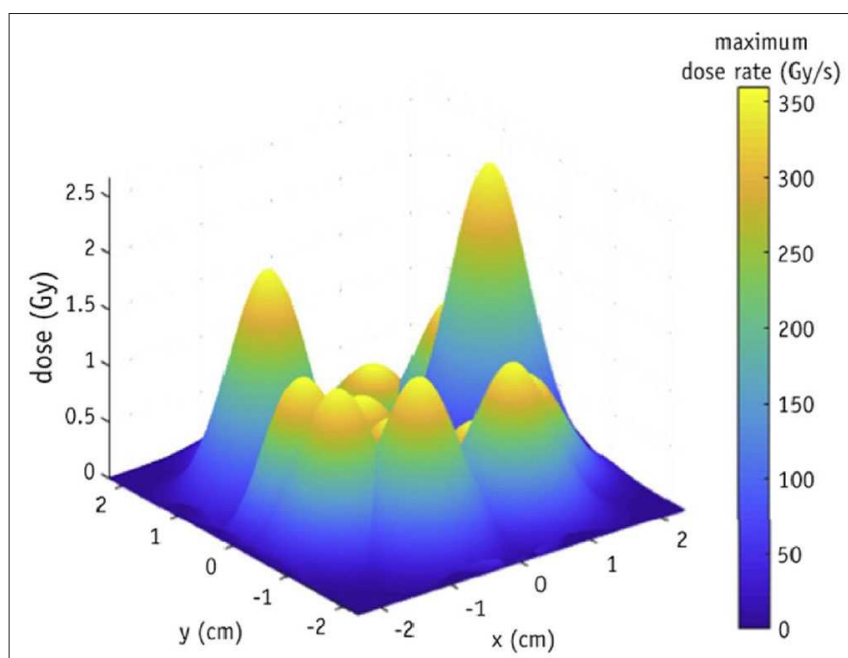

FIGURE 4 | Spatial distribution of dose-rate within a pencil beam scanned proton beam illustrating the role of scatter contribution from adjacent spots, which leads to an inhomogeneous distribution of dose-rate. A point detector or an imaging array with coarse detector spacing will be unable to measure dose-rate distribution with accuracy. Figure adapted from Marlen et al. [34]. Reprinted from "Bringing FLASH to the Clinic: Treatment Planning Considerations for Ultra high Dose-Rate Proton Beams", Vol 106/Issue 3, Figure 2A, Copyright (2020), with permission from Elsevier.

resolution and high bandwidth read-out methods are needed. For instance, some excellent dose-rate independent detectors like radiochromic film, alanine and TLD's etc. can only provide passive dose monitoring. Additionally, even though certain dosimeters can provide online dose-monitoring, they encounter other issues at ultra-high dose-rates which limits their capability. For example, clinical linear accelerators employ a monitor chamber in the gantry head which records machine output in real-time and serves as a beam-off signal when the recorded dose matches the required dose. However, most commercial ionization chambers start to show saturation or decreased ioncollection efficiency at high dose per pulse conditions rendering online monitoring of dose problematic at FLASH dose-rates. It has been shown by Jorge et al. [37] that despite correcting for ion-recombination effects at high doses per pulse, ICs can show deviations up to $15 \%$.

The minimum time resolution needed depends on the type of radiotherapy source used, or the purpose of the dosimetric measurement being performed. If the purpose is to perform field homogeneity tests or measure total dose, then real-time dose monitoring is not critical. On the other hand, if the machine output is to be investigated in real-time, a detector needs to be able to temporally resolve individual linac pulses at minimum. Most radiotherapy sources are pulsed with a repetition rate that varies depending on the source of particle acceleration used, spanning between $\sim 100 \mathrm{MHz}$ in cyclotron sources to $\sim 100 \mathrm{~Hz}$ in linac-based sources, which however can be broken down into $\sim \mathrm{GHz}$ bunching repetition rates. More importantly, the temporal resolution should be driven by the dynamics of underlying biological and chemical changes driving
FLASH effect, which itself is currently a topic being intensively investigated [38-41]. The time resolution requirement shall therefore be specified in terms of temporal division of machine output, which most closely matches the biochemical dynamics of FLASH.

\section{Dynamic Range}

Dynamic range of a dosimeter is another issue pertinent to dosimetry in FLASH. It has been shown that the oxygen depletion effect is highly dependent on the total dose delivered [38, 42, 43], with oxygen being depleted rapidly at high doses. If the oxygen depletion effect is indeed the underlying mechanism of the normal-tissue protection, it would be expected that preclinical studies will move toward hypo- or single-fractionated regimen with high doses per fraction to increase the therapeutic ratio. Currently, FLASH studies have been performed where total doses up to $40 \mathrm{~Gy}$ have been delivered in a short duration or in some cases single pulse of radiation. For in-vivo dose verification, this implies that a dosimeter is required which does not suffer from saturation effects and maintains a linear or otherwise predictable behavior in response to dose. Dynamic range in this case can be defined as saturation limit for one single pulse; a quantity related to the dose-rate linearity limit in Figure 2. (i.e., saturation limit would be the dose-rate linearity limit divided by a typical duration of a linac pulse). The dynamic range essentially translates to the maximum and minimum dose-delivered in a pulse that can be accurately measured. Radiation damage can also potentially be an issue for sensitive detectors such as MOSFET, however in most cases, the damage threshold for dosimeters is orders of magnitude higher compared to the dose threshold where dosimeters start showing saturation and non-linearity.

\section{Other Ideal Characteristics}

While, the previous few sections were primarily focused on dosimetric aspects unique to FLASH, there are still other characteristics that make certain radiation dosimeters ideal for dosimetry. These characteristics go hand in hand with the ones discussed previously because they will eventually lead to erroneous measurement of dose and subsequently dose-rate. Mainly, an ideal dosimeter should be tissue-equivalent and not perturb the radiation field so that it can serve as an accurate surrogate for dose measurement inside the patient. A parameter closely tied to tissue equivalence is the energy dependence of the dosimeter. Ideally, a dosimeter that is energy-independent is required, because one would want the detector to respond uniformly to irradiation irrespective of the radiation quality. For example, certain non-tissue equivalent detector can over respond when there is significant low energy scatter present in the radiation field leading to erroneous measurements. These issues are amplified at small fields and can cause significant errors. In general, correction factors for small fields can be divided in two categories: (1) volume averaging, (2) other non-ideal characteristics. While volume averaging is a purely geometrical concept and can be minimized by using dosimeters which are small in volume, non-ideal characteristics are harder to correct for. The volume averaging correction factor on the central axis is within $1-2 \%$ if the size of the detector is $1 / 4$ th of the diameter of 
the incoming beam [44]. For example, the unshielded Stereotactic Field Diode (SFD) diode ( $0.6 \mathrm{~mm}$ diameter) by IBA has a volume averaging correction factor of 1.003 for a $5 \mathrm{~mm}$ circular beam [45]. However, the overall correction factor for the diode has been typically found to be $<1$ in literature, which implies that the diode tends to over respond to radiation [46]. This over-response is explained by the presence of high- $Z$ silicon in the diode which offers an increased photoelectric absorption coefficient at lower energies. In FLASH, these non-ideal characteristics can be critical because of the use of small fields as discussed above.

\section{RADIATION DOSIMETERS}

\section{Charge Based Dosimeters}

Most charged based dosimeters are based on the principle of creation of ion pairs or charges which can be collected and correlated to dose. In Ionization Chambers (IC), the collection of ion-pairs is facilitated by an application of an external bias across the electrodes. The voltage applied is typically high enough, such that all liberated charges are collected. However, at high doses per pulse, ion-pairs can recombine before they are swept across the E field and collected by the electrodes. This can lead to a decrease in sensitivity with increasing dose per pulse. While ionrecombination effects can be accounted for at moderate doses per pulse using Boag's model [47], at high dose per pulse the model breaks down. However, it should be noted that certain commercial IC chambers, such as the Advanced Markus IC by PTW can efficiently collect ions at dose-rates as high as $\sim$ $300 \mathrm{~Gy} / \mathrm{s}$ and a dose per pulse of $\sim 5 \mathrm{mGy}$. This is sufficient for conventional flattening filter free beams and pencil beamscanned proton beams. Nevertheless, at high instantaneous doserates and doses per pulse typically used in FLASH, a correction factor would be needed to correct for ion-recombination. A study performed by Petersson et al. [48], looked into ionrecombination effects of the Advanced Markus IC with their FLASH setup. The authors came up with a model to account for ion-recombination and polarity effect for the aforementioned chamber (Figure 5). It can be seen that the ion-collection efficiency has a strong dependence on dose per pulse above $1 \mathrm{~Gy}$. With the correction factor applied, they concluded that the chamber could be used for FLASH dosimetry. In particular, they were able to measure a dose-per-pulse of $10 \mathrm{~Gy}$. Interestingly, FLASH studies conducted with protons have employed ICs for dosimetric verification but without application of any correction factor. For example, Patriarca et al. [18] employed a IC in their proton FLASH setup which used a cyclotron-based $230 \mathrm{MeV}$ (IBA) proton beam as the radiation source. With cyclotron-based sources, high pulse repetition rates of around $\sim 100 \mathrm{MHz}$ can be achieved with pulse duration of around $\sim 2 \mathrm{~ns}$. In this case, one can assume a $100 \%$ duty cycle, which drastically reduces the instantaneous dose rate or dose delivered in a pulse. Using Boag's method [47], the authors found that the total recombination of ions was around $1 \%$ at a maximum mean dose-rate of $80 \mathrm{~Gy} / \mathrm{s}$. Another study conducted by Beyreuther et al. [13] used a 224 $\mathrm{MeV}$ proton beam at a dose-rate of $100 \mathrm{~Gy} / \mathrm{s}$ and concluded that the pulse duration $(\sim 100 \mathrm{~ms})$ was an order of magnitude higher than the ion-collection time for the Advanced Markus chamber ( $\sim 10$ us).

Solid-state detectors such as diamonds and diodes have been used extensively for dosimetry in modern radiotherapy techniques due to their high sensitivity and small size. The operation of silicon diodes and diamond detectors is similar to IC chambers in that radiation produces electron-hole pairs which can be collected. Essentially, they can be thought of as solidstate ICs. Whereas, direct recombination is the dominant process in IC, charge recombination in solid state detectors is a more complex process dominated by indirect recombination, because of the presence of RG (recombination-generation) centers and impurities that can act as trap centers. In general, dose-rate dependence of solid-state detectors can be modeled as $\sigma \sim \mathrm{D}_{\mathrm{r}}^{\Delta}$, where $\sigma$ is the electrical conductivity, $D_{\mathrm{r}}$ represents the doserate and $\Delta$ is a fitting parameter which describes the doserate dependency of the detector [51]. Multiple studies have investigated the dose-rate dependence of diodes and diamonds. Ade et al. [52] found the fitting parameter $\Delta$ for some diamond detectors to decrease by as much as $9 \%$ when the dose-rate was increased from 2.25 to $3.07 \mathrm{~Gy} / \mathrm{min}$. Interestingly, diamond detectors have been have been reported to show increased or decreased sensitivity with increasing dose per pulse depending on their construction i.e., pure crystals, Chemical Vapor Deposition (CVD), high-pressure high-temperature HPHT [52]. While diamond detectors have not been extensively used for ultrahigh dose-rate experiments, a microDiamond detector type 60019 (PTW) was used for one proof-of-concept protonbased FLASH study by Patriarca et al. [18]. The sensitivity of the aforementioned diamond detector is shown in Figure 5. Recombination in diodes is also a complex physical process; whereas sensitivity of IC decreases with increasing dose per pulse, diodes are known to over respond at high dose per pulse [5355]. The physical basis of this dependence is due to insufficient number of RG centers available for the excess minority carriers to recombine at high dose-rates and doses per pulse. Therefore, a larger fraction of charges is left behind and can be collected by the electrode, leading to increased sensitivity of the diode. To the best of our knowledge, no FLASH study has used diodes for dosimetric verification. Kinetic modeling of the recombination process in solid-state detectors has been carried out by multiple groups and we point the reader toward those references for a deeper understanding $[49,54-56]$.

The time resolution of charged based dosimeters is mainly limited by the ion-drift velocity, mobilities of the different charge carriers present and other fundamental parameters such as the transit time (time taken for a charge to be completely collected) and minority carrier lifetime etc. For ICs with a typical external bias of $300 \mathrm{~V}$, the temporal resolution usually ranges from a few ms to hundreds of ms [57]. For indirect band gap semiconductors, such as silicon, the time-resolution can be on the order of a few ms [58]. Pure diamond detectors, due to their superior electron and hole mobilities, can offer time resolution on the order of a few ns [59], whereas, most synthetic diamonds (i.e., CVD based) have a minority carrier lifetime of a few us [60]. Therefore, real-time dose monitoring is indeed possible with diodes, diamonds, and IC. However, it 


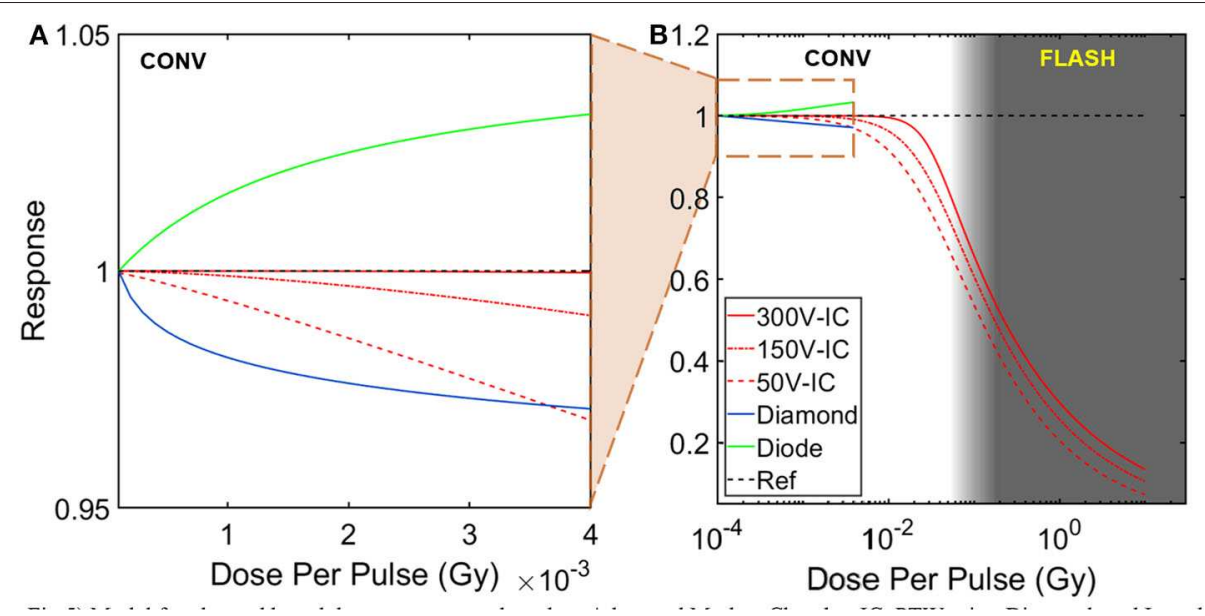

FIGURE 5 | Model for charged based detector response based on Advanced Markus Chamber IC, PTW microDiamond, and Isorad Gold (n-type) diode detectors. Dose per pulse dependency of detector response are shown for (A) conventional beams and (B) FLASH beams. Advanced Markus Chamber IC response (charge collection efficiency) was calculated for three different bias voltages and the only charge-based detector to be tested in FLASH dose rates [48]. Model for diamond detector response (charge collection efficiency) and diode detector response (sensitivity) were only tested at conventional dose rates [49, 50].

should be reiterated that the main limitation for such devices is the change in sensitivity, non-linearity, and saturation due to charge recombination at high instantaneous dose-rates relevant in FLASH. For pre-clinical FLASH studies, multiple authors $[19,61]$ have circumvented this issue by using a Faraday cup; a conductive metal cup which accumulates charge when put in the beam's path. The main advantage of using a Faraday cup is that saturation, ion-collection, and recombination effects can be avoided. Additionally, Faraday Cups have been used with nanosecond time resolution in studies conducted with high energy and highly pulsed charged particle beams [62-64].

Typically, measurements performed with charge based detector are point (1D) or planar measurements (2D). The advantage of solid-state charge based detectors over gas-filled ICs is that they offer superior spatial resolution because of their increased sensitivity to radiation. For instance, the PTW microDiamond detector, if used in edge-on configuration (i.e., smallest dimension normal to the incident beam), exhibits a resolution of $1 \mu \mathrm{m}$. Another high resolution charge-based dosimeter of interest is the silicon single-strip detector (SSD) which has been touted as a potential dosimetric tool for Microbeam Radiation Therapy. The SSD was used at the European Synchrotron Radiation Facility and has been shown to demonstrate very high spatial resolution $(\sim 10 \mu \mathrm{m}$ resolution) and high dynamic range [65]. Despite their high spatial resolution, one caveat of typical charge-based dosimeters is their energy dependence and tissue non-equivalence. This limits their usefulness in small field dosimetry which can lead to erroneous measurement of dose-rate in pre-clinical animal FLASH studies employing small beams.

\section{Chemical Dosimeters}

Certain materials that undergo structural changes, produce radicals, or change color when irradiated can be classified as chemical dosimeters. For example, when a solution of ferrous sulfate (Fricke) is irradiated, ferrous ions $\mathrm{Fe}^{2+}$ are oxidized to ferric ions, $\mathrm{Fe}^{3+}$. The number of ferric ions produced is proportional to dose delivered and can be quantified by measuring the optical density of the solution. Fricke dosimeter was used by Hendry et al. [61] in their study on effects of high dose-rate on oxygen concentration. However, diffusion of ferrous ions over time makes this technique sensitive to low dose-rates [66]. Similar in nature to the Fricke dosimeter, methyl viologen is another tool that was used by Favaudon et al. [29] in their FLASH setup for online monitoring of dose. Dosimetry was performed by optical detection of the $\mathrm{MV}^{\cdot+}$ radical at $603 \mathrm{~nm}$. The authors were able to monitor dose synchronously with the electron-pulses, but a decay in the $\mathrm{MV}^{+}+$radical with time (on the scale of a few minutes) was observed that ultimately led to loss in absorption; an issue which can play a major role at low dose per pulse/dose-rate conditions. Therefore, while, certain chemical dosimeters can provide absolute dosimetry and realtime detection of dose with $\sim$ ns resolution, the radiation induced species in these materials are generally not stable and can either diffuse spatially $\left(\mathrm{Fe}^{3+}\right)$ or decay with time $\left(\mathrm{MV}^{+}\right)$which makes such setups unsuitable for real-time dose monitoring in FLASH.

Fortunately, chemical dosimeters that produce stable radiation-induced species are available. One such dosimeter is Alanine and has been extensively used in preclinical FLASH studies [12, 37]. Alanine is an amino acid, which forms a stable free radical upon irradiation. The concentration of the free radical is proportional to the absorbed dose, which can be probed using an electron paramagnetic resonance (EPR) spectrometer. Alanine dosimeters exhibit a linear response over a large dynamic range (2 Gy-150 kGy) and are therefore routinely used in industrial facilities. Although, at doses below $2 \mathrm{~Gy}$, alanine can show considerable relative uncertainty of $\sim 1.5 \%$ [67]. However, this might not be an issue for FLASH 
dosimetry because generally high doses are needed to elicit the FLASH effect. The real value of alanine dosimeters for FLASH dosimetry is in their excellent dose-rate independence (up to $\sim 3$ $\times 10^{10} \mathrm{~Gy} / \mathrm{s}$ [68]). Recently, alanine was used at the European Synchrotron Radiation Facility (ESRF), which is capable of producing really high dose-rates ( $\sim 10 \mathrm{kGy} / \mathrm{s})[69]$; the response of alanine was found to agree well with the PTW PinPoint IC, when the latter was corrected for ion-recombination effects.

Perhaps the major advantage of certain chemical based detectors in their inherent ability to provide planar or $3 \mathrm{D}$ measurements. One really popular and perhaps the major workhorse of the radiation dosimetry world, is the polydiacetylene based self-developing radiochromic film. Upon irradiation, the film undergoes color change by polymerization. The change in color is typically quantified in terms of the optical density as measured by a densitometer or in some cases via microscopy. Radiochromic films can be considered to be the ideal dosimeter, in that they are energy independent, tissue equivalent, and demonstrate really high spatial resolution (submicron) limited only by the digitizing method. Additionally, dose-rate independence of radiochromic films is well-established in literature and they have been found to be independent of any dose-rate effects up to a dose-rate of $15 \times 10^{9} \mathrm{~Gy} / \mathrm{s}$ [70]. In fact, EBT3 Gafchromic Film (Ashland, Wilmington, DE) was used by Patriarca et al. [18] to evaluate dose-rate independence of other dosimeters used in their FLASH setup. A detailed study was conducted by Jaccard et al. [71] ${ }^{(\mathrm{p} 3)}$ on the suitability of radiochromic film for high dose-rate FLASH dosimetry with the Oriatron eRT6 electron linear accelerator (PMB-Alcen, Peynier, France) and they concluded that film was independent up to a $\dot{\mathrm{D}}_{\mathrm{p}}$ of $8 \times 10^{6} \mathrm{~Gy} / \mathrm{s}$. Additionally, radiochromic film has been used to measure dose homogeneity and verify field sizes in various different FLASH studies [9-11] and was also used to verify dose (along with alanine) for the human patient treated with FLASH. One of the major drawbacks of radiochromic film is that measurements are performed offline, typically $24 \mathrm{~h}$ post exposure to account for the fact that polymerization does not stop immediately after irradiation. In theory, real-time read-out of film can be performed with ms time resolution, since it has been reported in literature that polymerization is largely "complete" within $2 \mathrm{~ms}$ of a $50 \mathrm{~ns}$ pulse [72]; however, as started earlier, polymerization still continues post-exposure which can act as a confounding variable for near real-time dosimetry. Nonetheless, attempts have been made at real-time readout of radiochromic film $[73,74]$.

Certain chemical dosimeters can provide true $3 \mathrm{D}$ spatial dose distribution at high resolution and in patient geometry. This has been facilitated by the recent advent of gelatin-based polymers, which avoid the problem of ion diffusion encountered in Fricke dosimeters. However, diffusion in polymer gels can still occur in the first hour post irradiation and at high dosegradients and high doses [75]. Essentially, polymer gels act as $3 \mathrm{D}$ radiochromic film, except that the change in optical density is probed in $3 \mathrm{D}$ as opposed to a single 2D plane. Multiple methods have been used to probe these radiation sensitive gels, such as MRI, x-ray CT, ultrasound and optical projection tomography (OPT) [75]. However, OPT has stood out as the more popular read-out method, because of the high spatial resolution it offers. Unfortunately, like all chemical dosimeters, some change in signal is expected with time. Change in signal post-exposure coupled with the fact that complicated read-out machinery is needed to probe the response to radiation, renders this technique unsuitable for real-time dose measurement. More importantly, in FLASH context, polymer based gels have been known to show dose-rate dependence, which might be attributable to competing radiation induced chemical reactions in the gel and the doserate dependence of water radiolysis products [76]. The dose-rate dependence seems to be a function of concentration of oxygen scavengers in the matrix, with less dose-rate dependence seen at high concentrations of $\mathrm{O}_{2}$ scavengers [77]. Dose-rate dependence is also a function of the type of monomer unit of the gel [76]. For a more detailed analysis on the origin of dose-rate dependence in polymer gels, we refer the reader to a comprehensive review by De Deene et al. [76].

\section{Luminescent Dosimeters}

In this text, luminescence refers to any technique which utilizes generation of optical photons in response to radiation as a surrogate for dose. This generally includes thermoluminescent detectors (TLD), optically stimulated luminescence detectors (OSLD), organic/inorganic scintillators and Cherenkov radiation. Physical properties that enable luminescent detectors to be of value in FLASH will be discussed.

\section{TLD and OSLD}

When impurities are added to certain crystals, charge trapping occurs due to the added energy levels in the conductionvalence band gap. These additional energy levels act as traps for electrons and holes. Application of an external stimulus allows the trapped electrons and holes to escape allowing recombination at luminescent centers. It is this recombination process which leads to luminescence (Figure 6). Depending on the external stimulus, the dosimeters can be classified as TLD (thermo-luminescent dosimeter) or OSLD (optically stimulated luminescent dosimeter). The luminescence is considered to be delayed because the electrons and holes can remain trapped over long periods of time (sometimes up to thousands of years) and can only be read-out after stimulation. The practical implication of this is that real-time dosimetry is not feasible using TLD or OSLDs. In some cases however, certain materials such as europium-doped alkali halides, may exhibit short trap emptying $(\sim 25 \mathrm{~ms})$ and luminescent decay times $(\sim 1 \mu \mathrm{s})$ which may render real-time dose monitoring possible [78].

Despite the lack of real-time readout, TLDs and OSLDs are of great importance in high dose-rate dosimetry because of their excellent dose-rate independence. In fact, one of the earliest studies of dose rate effect on skin toxicity in mice (1980) by Inada et al. [79] used a lithium borate TLD to verify dose. They confirmed the lithium borate TLD to be independent up to a dose rate of $1.5 \times 10^{9} \mathrm{~Gy} / \mathrm{s}$. Dose-rate dependency of TLDs has been investigated by multiple authors over the last few decades. Karzmarck et al. [80] found LiF TLD to be dose-rate independent up to $2 \times 10^{6} \mathrm{~Gy} / \mathrm{s}$. Tochilin and Goldstien [81] found the same TLD to be dose-rate independent up to $1.7 \times 10^{8} \mathrm{~Gy} / \mathrm{s}$. More 

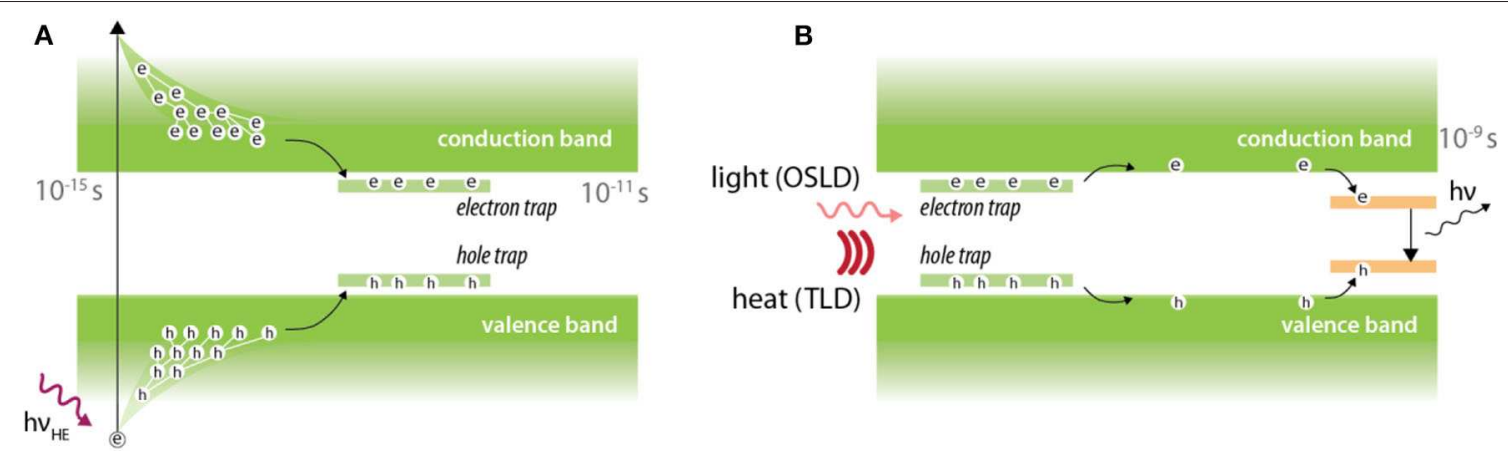

FIGURE 6 | Luminescence in thermally and optically stimulated luminescent detectors. (A) Irradiation leads to promotion of electrons from the valence band to conduction band. Electron and holes are subsequently trapped at trap centers which are introduced by doping impurities into crystals. (B) An external stimulus in the form of heat or light is then provided to facilitate electron-hole recombination at luminescent center, which leads to production of optical photons.

recently, Karsch et al. [70] compared dose-rate independence of various detectors including TLDs and OSLDs. They found TLD and OSLD to be dose-rate independent up to $4 \times 10^{9} \mathrm{~Gy} / \mathrm{s}$ within $2 \%$. In the context of FLASH, Jorge et al. [37] compared LiF-100 TLD (Thermo Fisher, USA) against two dose-rate independent dosimeters, alanine and radiochromic film. The Oriatron eRT6 linac was used for this study with dose-rates ranging from 0.078 Gy/s up to 1,500 Gy/s and the results are presented in Figure 7. Alanine, film, and the LiF-100 TLD were found to agree within $3 \%$ at all dose-rates.

In addition to dose-rate independence, TLD can be manufactured to be small and in powdered form. This is beneficial for small field dosimetry where high resolution is required. Additionally, the small form factor coupled with the ability to read out dose post irradiation renders TLDs as a viable tool for in-vivo dose verification. Indeed, for one FLASH study on mice whole brain irradiation [15], dosimetric verification was performed in-vivo using $3 \times 3 \times 1 \mathrm{~mm}^{3}$ TLD chips embedded inside the brain of a mouse cadaver at different points. A total dose of $10 \mathrm{~Gy}$ was either delivered in a single 1.8 us pulse or at conventional dose-rates $(0.1 \mathrm{~Gy} / \mathrm{s})$. The placement of the TLDs and the dose verification are shown in Figure 8. The dose measured by the TLDs agreed well with the prescribed dose of $10 \mathrm{~Gy}$. Additionally, no dose rate effect was seen between measurements performed at $0.1 \mathrm{~Gy} / \mathrm{s}$ compared to dose delivery in a single 1.8 us pulse $\left(5 \times 10^{7} \mathrm{G} / \mathrm{s}\right)$. This was one of the first non-superficial, in-vivo measurements performed at FLASH dose-rates.

One caveat of TLDs and OSLDs is that measurements are usually limited to a point. To overcome this, a few investigators have studied the possibility of using planar arrays of TLDs and OSLDs to measure spatial distribution of dose. One such TLD array was designed and tested at the European Synchrotron Radiation Facility (ESRF) by Ptaszkiewicz et al. [82]. The study was primarily aimed at Microbeam Radiation Therapy (MRT); a novel external beam radiotherapy technique in which quasiparallel beams with widths around $25-50 \mu \mathrm{m}$ separated by $100-$ $400 \mu \mathrm{m}$ are delivered at ultra-high dose rates. The results are relevant to FLASH because dose-rates in MRT can reach up to a few $\mathrm{kGy} / \mathrm{s}$ [33]. The TLD array consisted of LiF:Mg, Cu, P 10 x 10 $\times 0.3 \mathrm{~mm}^{3}$ foils with different grain sizes (up to $150 \mu \mathrm{m}$ ). Dose read-out was performed using a 12-bit CCD camera with submillimeter resolution. Therefore, dose-rate independence and sub-millimeter resolution make this setup an attractive choice for FLASH. Reusable 2D OSLD arrays have also been constructed with submillimeter resolution and large dynamic dose range $[83,84]$.

Another passive luminescent detector of note is the Fluorescent Nuclear Track Detector (FNTD) [85]. FNTDs employ a single crystal of Aluminum Oxide doped with Magnesium and Carbon $\left(\mathrm{Al}_{2} \mathrm{O}_{3}: \mathrm{C}, \mathrm{Mg}\right)$ with additional oxygen vacancy defects. The underlying physics is similar to OSLDs but with minor differences. Specifically, exposure to radiation produces new recombination or color centers which can then be probed non-destructively using microscopy techniques. In contrast, new recombination centers are not formed in OSLDs when exposed to radiation. This technique has been used for dosimetry in MRT [86] where spatial resolution of $1 \mu \mathrm{m}$ was achieved. Additionally, FNTDs have also been tested to be dose-rate independent up to $10^{8} \mathrm{~Gy} / \mathrm{s}$ and are capable of measuring dose over a large dynamic range ( $3 \mathrm{mGy}$ to $100 \mathrm{~Gy}$ ) [85]. Therefore, FNTDs are an attractive choice for dosimetry in FLASH.

\section{Scintillators}

Scintillation is the phenomena by which an interaction of certain material (scintillator) with high energy photon or charged particle results in emission of optical photons. Scintillators can be broadly divided into two different categories (1) organic and (2) inorganic [87, 88], with underlying physical mechanisms depicted in Figure 9. The process of scintillation in both material types follows a general mechanism composed of conversion, transport (migration), and luminescence. Organic scintillators are typically aromatic hydrocarbon compounds, that produce excited states by ionizing radiation, and subsequently luminesce due to allowed $\pi$ electron transitions between excited singlet state $S_{10}$ to various different vibrational sub-levels of the ground singlet state. When electronic transitions occur between singlet states, the 


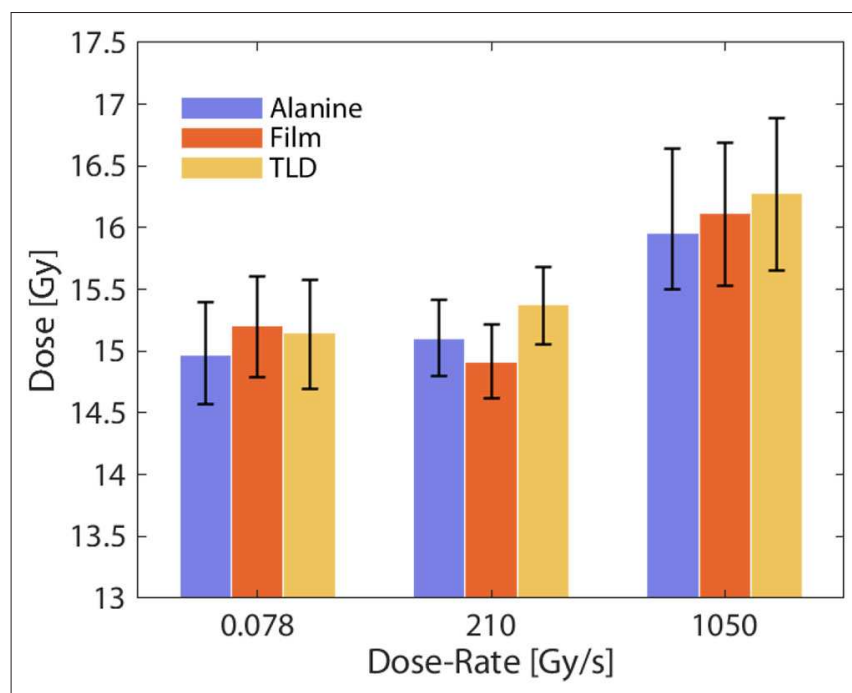

FIGURE 7 | Measured absorbed dose under different dose-rate conditions for alanine, film, and TLD. All detectors were found to agree within 3\% at conventional and FLASH dose-rates, indicating excellent dose-rate independence. Data adapted from Jorge et al. [37].

emission and decay of luminescence is on the order of a few ns. However, most organic solutions do not exhibit high scintillation efficiency and are therefore used in conjunction with a solute. In this case, energy transfer occurs mainly via solvent-solvent interactions, and ultimately to the solute by dipole-dipole energy transfer [90]. In the case of polar solvent-based scintillators (e.g., quinine solution in water), ions and radicals are formed rather than excited states, and therefore ion recombination must occur prior solute excitation [91].

Inorganic scintillators typically consist of single or polycrystalline materials, often doped with impurities that can act as luminescent centers. In the initial conversion phase, a large number of excited electrons and holes is created upon interaction of high energy photon or charged particle with the scintillator matrix, followed by thermalization and transport of created excited states to a luminescent center. Unlike in OSLD, an external stimulus to facilitate the release and recombination of electrons and holes is not needed due to the presence of an allowed transition at luminescent centers, and minimal number of charge traps. Similar to organic scintillators, rise and decay times for inorganic scintillators can also be on the order of a few ns.

Owing to their excellent tissue-equivalence and the ability to be miniaturized, multiple investigators have recommended the use of organic scintillators for small field dosimetry [21, 45, 92]. In particular, organic scintillators, such as the commercially available Exradin W1 (Standard Imaging) can be used as reference detectors for small fields against which correction factors for other detectors can be derived [45]. In contrast to organic type, inorganic scintillators are usually made with high- $Z$ materials and are therefore not tissue-equivalent; a scenario which need to be accounted for in radiation dosimetry. Nonetheless, they have a role to play in FLASH. Fast rise and decay times, radiation hardness and high detection efficiency due to increased photoelectric cross section for x-rays, makes inorganic scintillators an ideal tool for applications where superior time-resolution is required.

Typically, measurements performed with scintillators can be either point, planar 2D or 3D measurements. For point measurements, the setup usually consists of a small scintillator coupled to an optical fiber and a photodetector. Recently, Archer et al. [93] demonstrated the use of miniature BG400 plastic scintillator $(10 \mu \mathrm{m}$ thick) coupled to a fiber optic and a SiPM for dosimetry at the Imaging and Medical Beam-Line at the Australian Synchrotron with an average dose-rate of 4,435 Gy/s, resolving beams of $50 \mu \mathrm{m}$ width.

In case of planar or 3D measurements, the setup typically consists of a scintillating volume imaged remotely at high spatial resolution (sub millimeter) with a CCD or a CMOS camera. The prompt emission of light, coupled with high frame-rate imaging capabilities of modern imaging sensors, make this technique suitable for online monitoring of machine output and dose delivery under FLASH irradiation conditions. Optical imaging of scintillation using cameras during external beam radiotherapy has already been widely implemented [88, 94, 95]. In a recent study [96], a time-gated intensified CMOS camera was used to image complex stereotactic radiosurgery (SRS) plans at high dose-rates in a radioluminescent phantom. The authors were able to resolve complex and highly modulated dose distributions spatially and temporally. Due to its high spatio-temporal resolution, optical imaging has also been used for quality assurance purposes in pencil beam scanning (PBS) proton therapy; a technique which also utilizes high dose rates (up to $200 \mathrm{~Gy} / \mathrm{s}$ near the Bragg Peak). For example, Vigdor et al. [97] used a xenon gas scintillator coupled to large PMTs for monitoring $2 \mathrm{D}$ beam characteristics in real-time for pulsed and pencil beam scanning proton radiotherapy treatments. The authors demonstrated a spatial resolution of a few hundred microns. Additionally, they noted that the gas scintillator was able to measure up to a dose rate of $350 \mathrm{~Gy} / \mathrm{s}$, whereas an ionization chamber started exhibiting ion-recombination effects at much smaller dose-rates. In another study, Darne et al. [98, 99] used three CMOS cameras to image proton pencil beam scanning inside a phantom filled with a liquid scintillator. The authors were able to image at 91 frames per second with sub millimeter resolution. More recently, Rahman et al. [35] were able to resolve spatio-temporal (10 $\mathrm{ms}$ and $1 \mathrm{~mm}$ resolution) dose-rate dynamics up to $26 \mathrm{~Gy} / \mathrm{s}$ for proton PBS using a scintillating sheet and a CMOS camera. As shown in Figure 10, the imaging technique visualized the proton beam parameters that modulated dose rate distributions and introduced cumulative dose rate histograms that can potentially be used for optimizing dose rate distributions for patient planning in FLASH. One FLASH study by Favaudon et al. [29], used a 2D scintillating array coupled to a CCD camera ( $\operatorname{Lyn} x^{\circledR}$, IBA) for monitoring beam profiles. The scintillating screen was a $0.5 \mathrm{~mm}$ thick gadolinium based plastic material with an active area of $300 \times 300 \mathrm{~mm}^{2}$ and a spatial resolution of $0.5 \mathrm{~mm}$. The detector was primarily used to assess field size, field homogeneity and dose linearity of the system and exhibited excellent linearity with increasing 

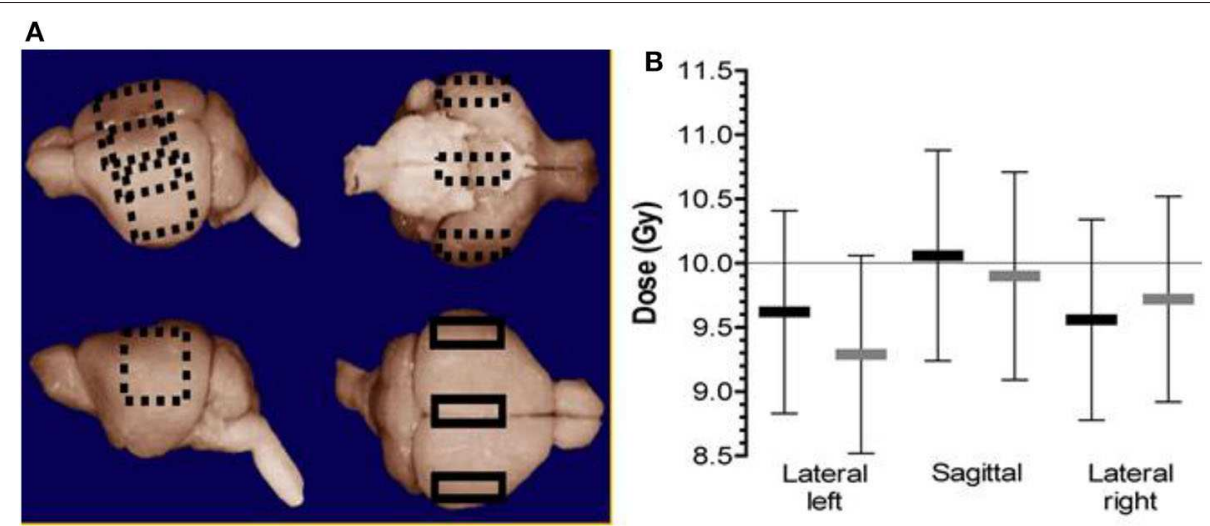

FIGURE 8 | (A) The three different positions where the TLDs were placed inside the brain of a mouse cadaver (sagittal/center, lateral left, and right). (B) The measured dose at different points. The black markers represent 10 Gy delivered in a single pulse of 1.8 us (FLASH). The light gray markers represent the dose delivered at a dose-rate of $0.1 \mathrm{~Gy} / \mathrm{s}$ (i.e., conventional dose-rate). Error bars represent the relative uncertainty in the absorbed dose measurements ( \pm 8.2\% in each case) [15]. Reprinted from "Irradiation in a flash: Unique sparing of memory in mice after whole brain irradiation with dose rates above 100 Gy/s," Vol 124/Issue 3, Figure 1, Copyright (2017), with permission from Elsevier.
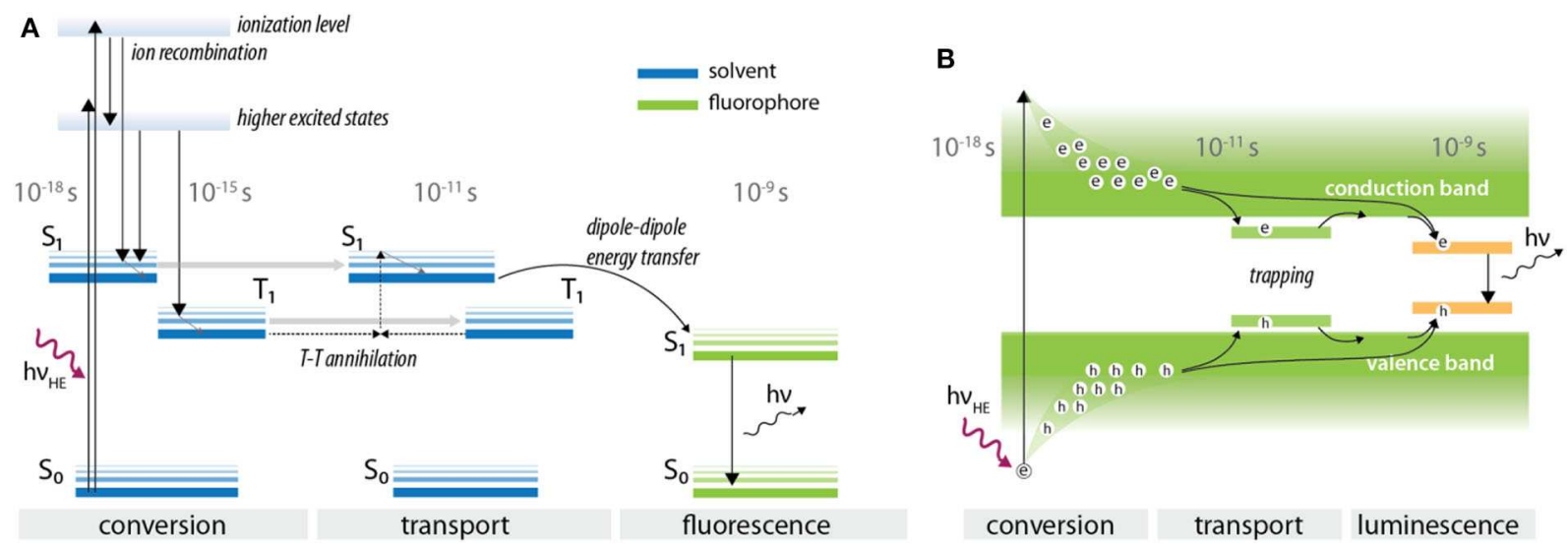

FIGURE 9 | Typical processes of scintillation in (A) organic and (B) inorganic scintillators. The time scales over which these processes occur are also shown, indicating excellent temporal resolution. (B) was adapted from Nikl et al. [89].

dose. The field homogeneity and FWHM values measured by the scintillating detector were within $1 \%$ at high and low dose rates. Peak dose-rate used in the study was around 2.4-3.5 $\times$ $10^{6}$ Gy/s. The Lynx ${ }^{\circledR}$ detector was also used by Beyreuther et al. [13] for measuring field homogeneity in their proton FLASH setup. Multiple investigators have now used cameras and scintillation to reconstruct dose in 3D [100-103]. In the context of FLASH, this implies that a dose-rate independent detector that can measure dose in $3 \mathrm{D}$ with high spatial and temporal resolution ought to be able to measure dose-rate distribution in $3 \mathrm{D}$ in real-time. This information can potentially be used to predict the spatial distribution of the protective effect of FLASH in patient geometry.

\section{Cherenkov Radiation}

Cherenkov radiation is the emission of optical photons in a dielectric medium when a charged particle travels at a phase velocity that exceeds the phase velocity of light in the medium. Electromagnetic fields associated with a charged particle can polarize the medium. If the particle moves slowly (relative to the speed of light in the medium), the relaxing dipoles experience a net destructive interference, and no light is emitted. However, if the particle's phase velocity exceeds that of light, asymmetric polarization can occur along the particle's trajectory, and the relaxing dipoles radiate energy with a net constructive interference, observed as visible Cherenkov radiation (Figure 11A). In contrast to scintillation, the Cherenkov light is not emitted isotropically, but rather in a cone with its axis aligned with particle trajectory. It has been shown by multiple groups that above the threshold for Cherenkov (261 keV in water) generation, the intensity of light emitted is proportional to dose [104, 105], albeit with prominent energy dependence for particles below $\sim 1 \mathrm{MeV}$. Importantly, Cherenkov light is created instantaneously [106] $\left(\sim 10^{-12} \mathrm{~s}\right)$ 

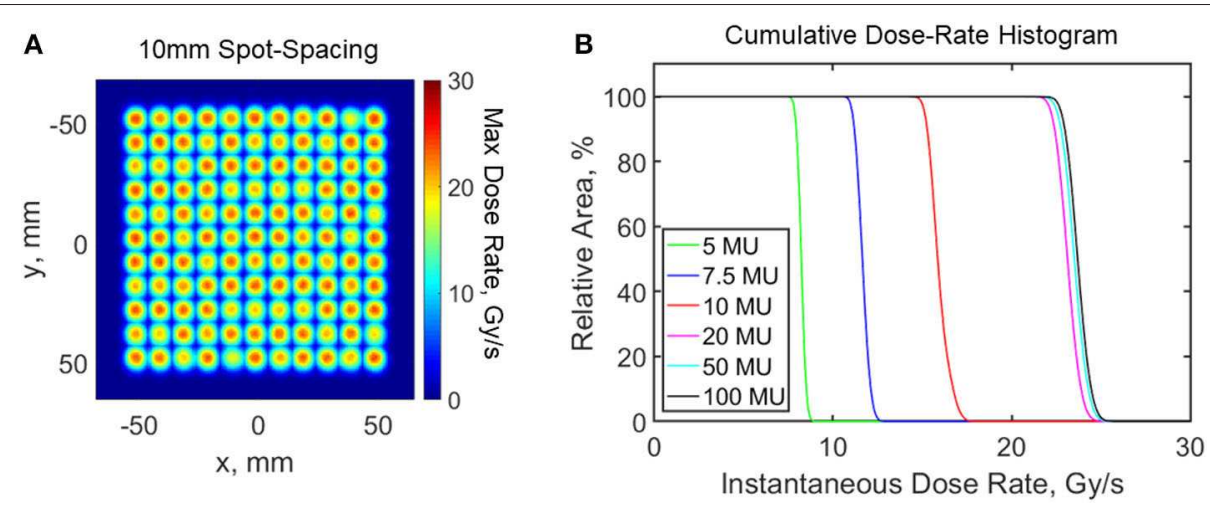

FIGURE 10 | Scintillating sheet imaged dose rates from a proton pencil beam scanning system with modulated beam parameters in the treatment plans. (A) Maximum dose rate distribution with $10 \mathrm{~mm}$ spot-spacing. (B) Cumulative dose rate histogram for varying minimum spot weight of a treated layer. Data adapted from Rahman et al. [35].
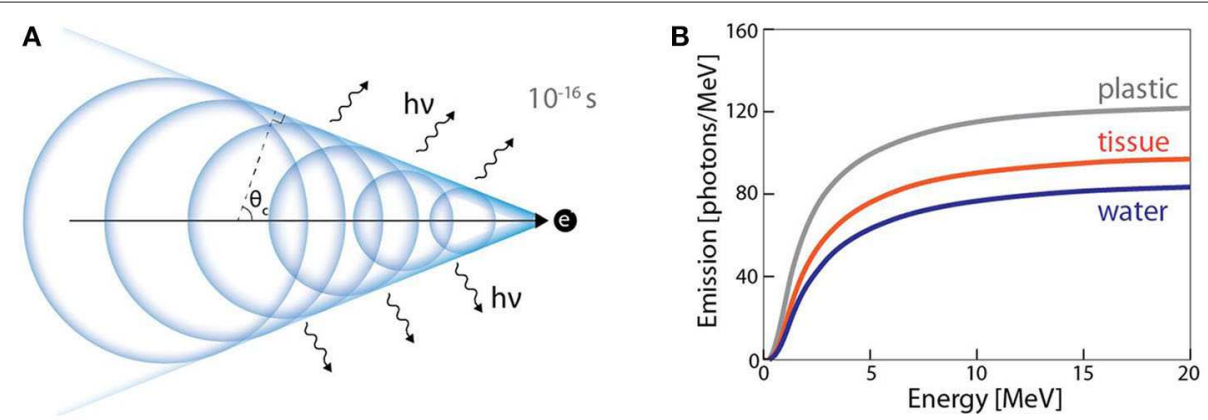

FIGURE 11 | (A) Huygens representation of Cherenkov radiation mechanism in dielectric medium. Light is generated in a cone at caustic angle $\theta_{\mathrm{C}}$ around the trajectory of charged particle. (B) Energy dependence of Cherenkov radiation for different materials (adapted from Glaser et al. [104]).

upon interaction of the charged particle with the dielectric medium; this is faster than what most scintillators are capable of because of the various non-radiative mechanisms specific to the process of scintillation. Multiple investigators have now made use of Cherenkov radiation as time-of-flight PET detectors [106-108] due to its fast time response. Cherenkov radiation has also found use in pulse radiolysis studies with pico [109] and femtosecond [110] time resolution. Additionally, Cherenkov radiation has been imaged in real-time [111] (p), [112] during multiple clinical radiotherapy treatments. The prompt nature of light emission, along with dose linearity makes Cherenkov emission an ideal tool for real-time dose monitoring. The general experimental setup for Cherenkov based dosimetric imaging is similar to the ones discussed earlier for scintillation dosimetry; an undoped optical fiber (i.e., production of Cherenkov and no scintillation) coupled to a photodetector or a volume capable of producing Cherenkov radiation imaged remotely with a camera. In the latter case, if a water phantom is subjected to radiation, Cherenkov emission can then be considered to be a water-equivalent dosimeter. However, due to inherent threshold below which no Cherenkov photons are generated, Cherenkov based detectors are expected to be energy dependent; a scenario which is not ideal for radiation dosimetry (Figure 11B).
In the context of FLASH, a Cherenkov probe was used for online monitoring of dose by Favaudon et al. [29]. A number of tests were performed to confirm the efficacy of the Cherenkov detector. In one of the tests, a single $1 \mu$ s pulse of 3.9 or $5.0 \mathrm{MeV}$ electrons were delivered to the probe. The area under the signal detected by the PMT (voltage against time) was found to be proportional to the energy of the beam. In another test, single pulses were delivered with increasing pulse widths $(0.1-2.2 \mu \mathrm{s})$, which essentially translates into changing dose. The authors noted that the integral Cherenkov emission increased with beam energy, pulse duration and dose, without any saturation effects. Based on these results, the authors concluded that Cherenkov radiation has potential to be a useful tool for online-dose monitoring under high and low doserate conditions.

\section{BIOLOGICAL EFFECTS AND DOSIMETRY: OER AND LET}

A complete overview of the radiobiological underpinning of the FLASH effect is outside the scope of this study. However, the role of oxygen depletion will be briefly discussed here, since 

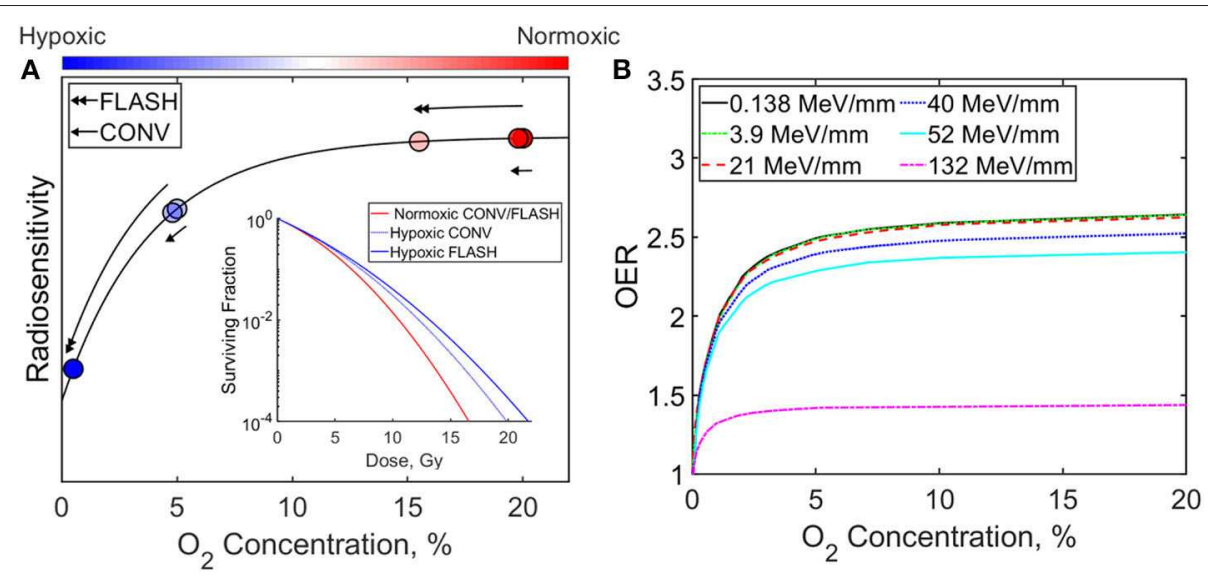

FIGURE 12 | (A) Radiosensitivity and cell surviving fraction dependency on oxygen concentration for FLASH and conventional (CONV) radiation therapy. Models are from Pratx et al. and Adrien et al. for normoxic (20\% oxygen concentration) and hypoxic (5\% oxygen concentration) cells. (B) Oxygen enhancement ratio with $\mathrm{O}_{2}$ concentration for irradiation with different LET [38, 113-115].

it is considered to be one of the major factors mediating the FLASH effect. Presence of molecular oxygen is known to make cells more susceptible to damage by radiation, as shown in the radiosensitivity curve in Figure 12A. This can be defined in terms of the oxygen enhancement ratio (OER), which is the ratio of dose needed to achieve the same biological effect under hypoxic and normoxic conditions. At ultra-high dose rates, it is hypothesized that transient hypoxia occurs which confers a protective effect on normal tissue. The improved differential response between tumor and normal tissue arises because the microenvironment surrounding solid tumors is already hypoxic [116] and remains largely unaffected by the depletion of oxygen. The time-scales over which oxygen depletion and reoxygenation occurs is important, since the underlying assumption is that oxygen is depleted at a rate faster than it can diffuse back into the normal tissue. Adrian et al. [113] in vitro study supports the model and their results indicated there was no difference between cell death in hypoxic (5\% oxygen concentration) cells, however cells under normoxic (20\% oxygen concentration) oxygen conditions, showed increased survival from FLASH compared to conventional irradiation [113]. This can be attributed to larger gradient in radiosensitivity at normoxic oxygen concentration, thus a more prominent FLASH effect.

Luminescence imaging, in addition to dose and dose rate, can measure oxygen concentration and play an important role in testing the hypothesis in vivo. There are many indirect methods of estimating oxygen concentration in tissue including quantifying vascular parameters (intercapillary distance, distance from tumor cells to nearest vessel), perfusion, gene expression, protein levels, metabolism, DNA damage [117]. However, there are only a few direct methods of measuring oxygen concentration or tension directly, including the standard procedure of using a polarographic needle electrode system [118-121]. Collingridge et al. [122] compared the standard polarographic method to an oxygen sensing system based on a time-resolved luminescence optical probe. The method relied on measuring the lifetime of the luminescence molecules from oxygen-quenching in the tip of the optical fiber and relating it to oxygen concentration. The authors confirmed that the time-resolved luminescence probe had the same degree of accuracy as a polarographic electrode system in measuring oxygen concentration. However, probes are invasive, measure at single points, and are scanned across the tissue to provide a histogram of oxygen concentration. Imaging techniques provide methods of quantifying oxygen concentration distributions. Positron emission tomography (PET) with $\mathrm{F}^{18}$ labeled markers has been used to image hypoxia, but scans may take $2-4 \mathrm{~h}$, which is much longer than the time scale of FLASH effects [117]. Alternatively, $\mathrm{F}^{19}$ based oximetry and magnetic resonance imaging can provide oxygen concentration at the multiple pixel level [123]. Electron paramagnetic resonance spectroscopy and imaging with oxygen sensitive particulate or water-soluble probes can also provide direct measurement of $\mathrm{pO} 2$ or $[\mathrm{O} 2]$ with sub-millimeter spatial resolution and temporal resolution $<<1$ s $[124,125]$. More recently, Cherenkov excited luminescent imaging (CELI) provided in vivo oxygen pressure maps based on lifetime imaging of fluorophore platinum(II)G4 (PtG4) [126, 127]. The Stern-Volmer equation was used to relate the decay of PtG4 to oxygen tension in tumors and normal tissue (pre and post euthanasia) (Figure 13). The imaging technique achieved submillimeter resolution of $\mathrm{pO}_{2}$ across the surface and near sub-surface of tissue and can potentially image $p \mathrm{O}_{2}$ post irradiation from a FLASH beam. This could be used to help relate the dose rate distribution to the oxygen depletion distribution in the tissue and effects on the OER.

The ability of certain luminescent based detectors to quantify linear energy transfer (LET) can also play a crucial role for FLASH. The OER is dependent on particle type and LET as shown in Figure 12B [114]. Heavy charged particles such as protons and alpha particles can have higher LET than photons or electrons and can reduce the effects of the OER. Thus, the oxygen depletion hypothesis brings into question whether 

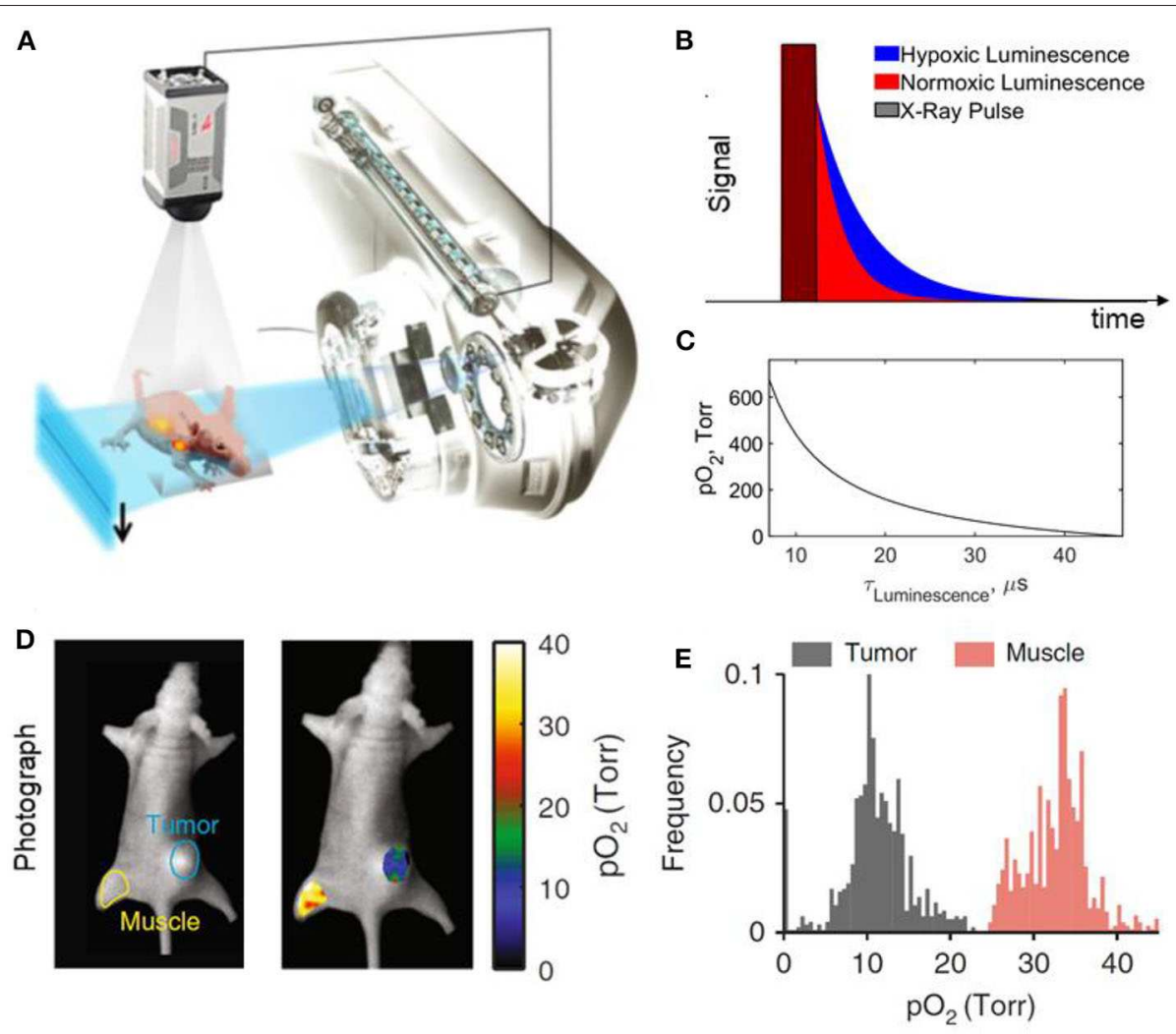

FIGURE 13 | Cherenkov excited luminescence imaging of oxygen concentration; (A) Schematic of a 6MV x-ray beam from the linear accelerator used to excite luminescence and captured by a time-synchronized camera; (B) Time signatures of the x-ray pulse and the imaged luminescence in hypoxic and normoxic tissue; (C) Relation between $\mathrm{pO}_{2}$ pressure and luminescent lifetime based on Stern-Volmer model; (D) $p \mathrm{O}_{2}$ map of muscle and tumor tissue with their respective histograms shown in (E). Figure adapted from Zhang et al., Bruza et al., and Cao et al. [127-129].

heavy charged particles will provide the same degree of FLASH effects as electrons and photon FLASH beams. Furthermore, LET distribution of heavy charged particles are not homogeneous and have a drastic increase at the Bragg peak of the beam. So, quantifying spatial distribution of the LET may be important in describing the differences in FLASH effects of heavy particles and photons/electron beams. Currently, Monte Carlo methods or analytical methods are often used to determine LET distribution for treatment plans $[130,131]$. However, only certain detectors can measure LET of charged particle beams and majority of them are based on luminescent techniques. Fluorescence nuclear track detectors (FNTD) have been used to measure LET of individual proton tracks $[85,132]$. However, FNTDs have a limited range of LET it can detect $(5-1,000 \mathrm{MeV} / \mathrm{mm})$, which does not include the range of LET distribution of proton beams. Alternatively, OSLD/TLD response dependency on LET can be utilized to determine both LET and dose distribution [133, 134]. Nonetheless, these methods are passive and do not quantify dose or LET distribution in real time. Alsanea et al. [135] showed that variable LET scintillation quenching in two different tissue equivalent organic scintillators can be utilized to determine dose and LET distribution in real time [135]. This method relies on Birk's law of scintillation quenching, requires a large difference in the quenching parameter, and requires the scintillators to be made of the same material (i.e., electron density). To note, silicon detectors have also been used to determine the mean LET of the proton and show its dependency on clinical proton beam energies (1-194 MeV) [136].

\section{DISCUSSION AND CONCLUSION}

A comprehensive list of dosimeters used in FLASH studies and other high dose-rate modalities is given in Table 1. The different columns represent some of the major issues identified in section Dosimetric Aspects of FLASH That Need to be Considered. The values are based on typical values and usage encountered in literature. Exception to these values do exist; for example, radiochromic film is categorized as a passive detector, but attempts are real-time dosimetry with film has been made in the past $[73,74]$. The "Measurement Type" column has bold entries in it, which indicate the way those dosimeters were employed in FLASH studies. The time resolution values are based on the underlying physics of the dosimeters, as explained previously. This does not take in to account the available bandwidth of the read-out method. Of course, the dead-time of the read-out electronics should be considered while dealing with such dose-rates. While some of these issues are not necessarily 
TABLE 1 | Dosimeters and their capabilities rated for potential FLASH dose measurement of key parameters.

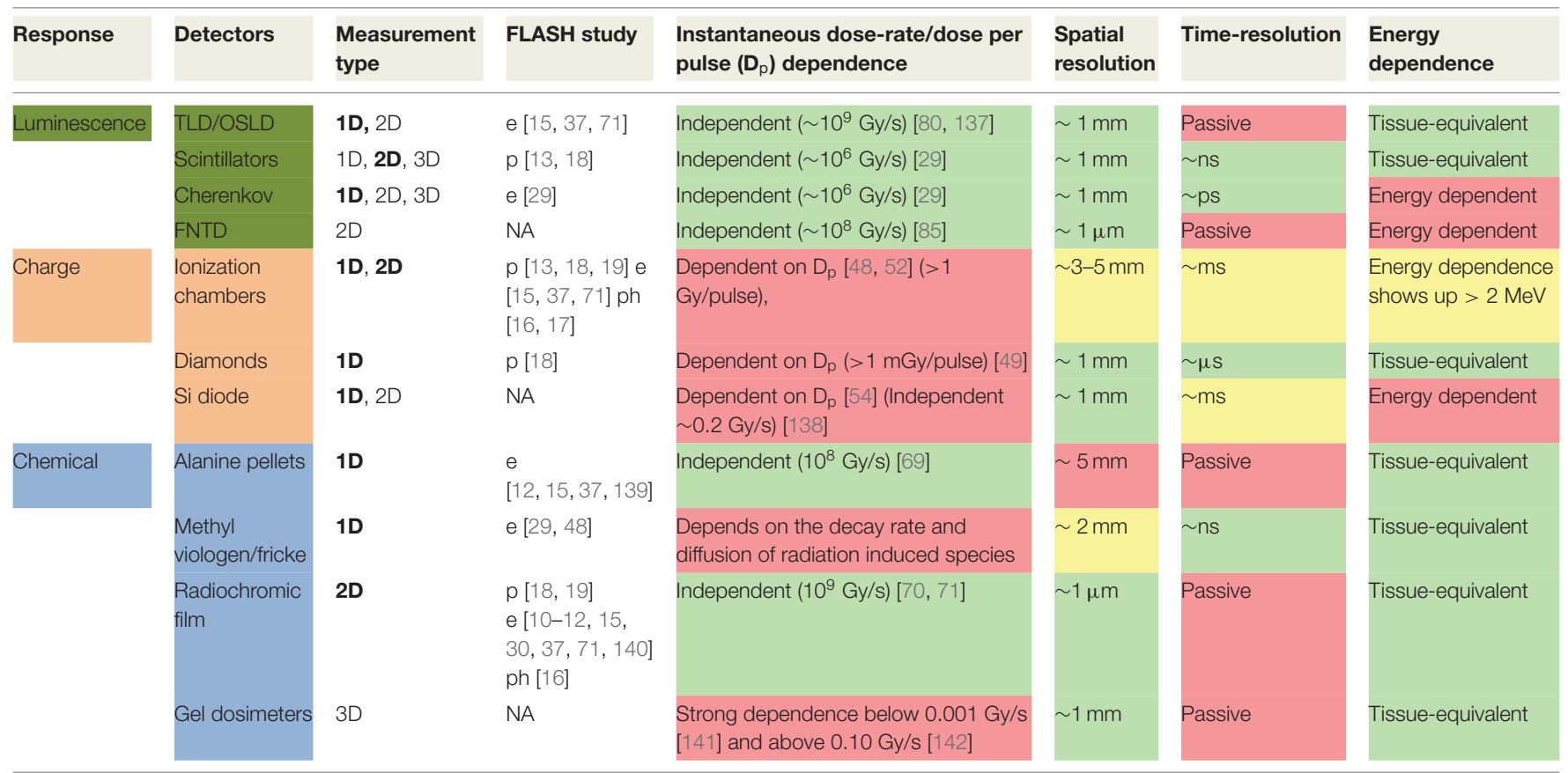

The color scheme of the "Response" and "Detectors" panel matches the spider plots in Figure 14. Performance of each dosimeter for a specific parameter is color coded: green (good), yellow (moderate), and red (poor).

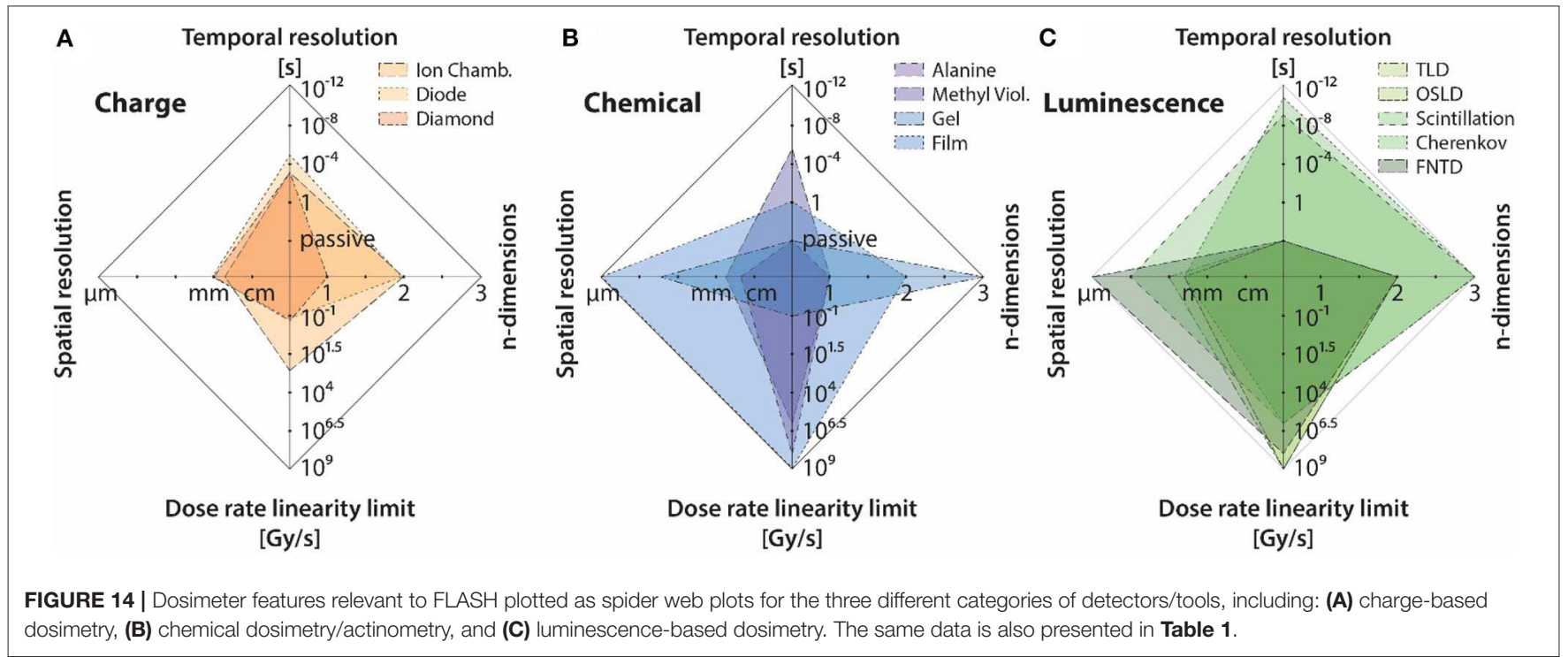

unique to FLASH, they nonetheless contribute to the overall dosimetric uncertainty.

Based on the unique temporal beam characteristics of FLASH, dose-rate dependence, spatial resolution (in particular, accurate measurement of spatial distribution of dose-rate in a broadbeam), and time-resolution parameters shall be emphasized. To compare detectors based on these parameters (and the number of dimensions it has measured up to in literature), a spider chart in presented in Figure 14, where (a), (b), and (c) refer to the three different categories of detectors. It can be seen that all luminescence based dosimeters exhibit excellent dose-rate independence. For chemical dosimeters, radiochromic film and alanine dosimeters also show dose-rate independence up to $10^{9} \mathrm{~Gy} / \mathrm{s}$. Even though methyl viologen is shown to be dose-rate independent up to a high dose-rate in Figure 14, they tend to be dose-rate dependent at really low dose-rates or low doses per pulse conditions because of the diffusion/decay of radiation induced species with time. Therefore, such chemical dosimeters, while promising at high dose-rates, might not be suitable if they are to quantify 
an in-homogenous distribution of dose-rate. Charged based dosimeters tend to have a complex dependence on dose rate and are highly dependent on the temporal characteristics of the beam.

For measurement of dose in real-time, it can be seen that, luminescent detectors again tend to be superior when compared to chemical and charge based detectors. Most scintillator based detectors provide $\sim$ ns resolution, whereas Cherenkov radiation in this regard provides the best theoretical time-resolution $(\sim p s)$; a fact which makes it an ideal candidate for onlinemonitoring of machine output without suffering from issues such as saturation or dose-rate dependence. Based on results presented by Favaudon et al. [29], it can be argued that Cherenkov detectors can play a role similar to that of monitor chambers in conventional radiotherapy. Other luminescent detectors, such as TLD/OSLD and FNTD are suitable for passive measurements. Nonetheless, they still have a role to play in FLASH, because of their dose-rate independence. Most chargebased dosimeters also offer decent temporal resolution; however, they are limited by their dependence on dose per pulse/doserate. Chemical based dosimeters tend to be feasible only for offline measurements, due to cumbersome read-out methods and the general instability (temporal and spatial) of radiation induced species.

Due to an increase in use of small radiation fields, detectors have been miniaturized to the extent, such that a resolution of $\sim 1 \mathrm{~mm}$ is achievable with most modern detectors. It is important to distinguish between spatial resolution of point detectors from that of imaging detectors. For point detector, the spatial resolution is defined in terms of the spatial extent of the sensitive volume. For imaging detectors, the interdetector spacing is perhaps a more suitable measure of spatial resolution. While, point solid state detectors, such as diamonds and diodes can indeed be constructed to be small, imaging arrays based on these detectors typically tend to exhibit an inter-detector spacing of 3-5 mm. Dose-rate dependence coupled with sparse detector spacing, makes these imaging arrays unsuitable for FLASH purposes. Additionally, solid-state devices tend to be non-tissue equivalent and energy dependent which can further complicate dosimetry due to small field issues.

\section{REFERENCES}

1. Chang DS, Lasley FD, Das IJ, Mendonca MS, Dynlacht JR, (editors). Therapeutic ratio. In: Basic Radiotherapy Physics and Biology. Cham: Springer International Publishing (2014). p. 277-82.

2. Hornsey S, Bewley DK. Hypoxia in mouse intestine induced by electron irradiation at high dose-rates. Int J Radiat Biol Relat Stud Phys Chem Med. (1971) 19:479-83. doi: 10.1080/09553007114550611

3. Hall EJ, Brenner DJ. The dose-rate effect revisited: radiobiological considerations of importance in radiotherapy. Int J Radiat Oncol Biol Phys. (1991) 21:1403-14. doi: 10.1016/0360-3016(91)90314-T

4. Town CD. Radiobiology. Effect of high dose rates on survival of mammalian cells. Nature. (1967) 215:847-8. doi: 10.1038/215847a0

5. Prempree T, Michelsen A, Merz T. The repair time of chromosome breaks induced by pulsed $\mathrm{x}$-rays on ultra-high dose-rate. Int J Radiat Biol Relat Stud Phys Chem Med. (1969) 15:571-4. doi: 10.1080/09553006914550871
Comparing chemical and luminescent detectors, it can be seen that radiochromic film provide the best possible spatial resolution. High spatial resolution, tissue equivalence and doserate independence make radiochromic films an ideal tool for measuring spatial distribution of dose-rate in FLASH. However, luminescent based detectors based on optical imaging techniques can provide the aforementioned qualities of radiochromic film, with the added advantage of high temporal resolution which makes real-time dose monitoring possible.

In addition to traditional dosimetry, the bio-chemical dose response of FLASH was also discussed. In particular, it was shown that luminescent techniques can sense oxygen tension in realtime and can also measure dose and LET simultaneously for particle therapy. These parameters are crucial to understanding the underlying radiobiological mechanisms of the protective effect of FLASH. Questions such as how the FLASH effect varies with LET, oxygen concentration etc. can be answered using these techniques. In conclusion luminescence was presented as a tool which can play a diverse role in the performing dosimetry and understanding the FLASH effect caused by ultra-high dose-rates.

\section{AUTHOR CONTRIBUTIONS}

$\mathrm{PB}$ and $\mathrm{BP}$ conceived the presented review paper, edited the manuscript, and created some figures. MA and MR took the lead in all research and writing the manuscript and making figures. RZ, DG, and BW contributed to feedback by editing and critical analysis of the manuscript. All authors contributed to the article and approved the submitted version.

\section{FUNDING}

This work was funded by the National Institutes of Health grants R01 EB024498, R01 EB023909, and Norris Cotton Cancer Center Core finds P30 CA023108, as well as the Thayer School of Engineering at Dartmouth.

\section{ACKNOWLEDGMENTS}

This manuscript has been released as a pre-print at [143].
6. Nias AH, Swallow AJ, Keene JP, Hodgson BW. Effects of pulses of radiation on the survival of mammalian cells. Br J Radiol. (1969) 42:553. doi: 10.1259/0007-1285-42-499-553-b

7. Berry RJ, Hall EJ, Forster DW, Storr TH, Goodman MJ. Survival of mammalian cells exposed to $\mathrm{x}$ rays at ultra-high dose-rates. $\mathrm{Br} \mathrm{J}$ Radiol. (1969) 42:102-7. doi: 10.1259/0007-1285-42-494-102

8. Watts ME, Maughan RL, Michael BD. Fast kinetics of the oxygen effect in irradiated mammalian cells. Int J Radiat Biol Relat Stud Phys Chem Med. (1978) 33:195-9. doi: 10.1080/09553007814550091

9. Favaudon V, Caplier L, Monceau V, Pouzoulet F, Sayarath M, Fouillade $\mathrm{C}$, et al. Ultrahigh dose-rate FLASH irradiation increases the differential response between normal and tumor tissue in mice. Sci Transl Med. (2014) 6:245ra93. doi: 10.1126/scitranslmed.3008973

10. Levy K, Natarajan S, Wang J, Chow S, Eggold J, Loo P, et al. FLASH irradiation enhances the therapeutic index of abdominal radiotherapy in mice. bioRxiv [Preprint]. (2019). doi: 10.1101/2019.12.12.873414 
11. Simmons DA, Lartey FM, Schüler E, Rafat M, King G, Kim A, et al. Reduced cognitive deficits after FLASH irradiation of whole mouse brain are associated with less hippocampal dendritic spine loss and neuroinflammation. Radiother Oncol. (2019) 139:4-10. doi: 10.1016/j.radonc.2019.06.006

12. Vozenin M-C, De Fornel P, Petersson K, Favaudon V, Jaccard M, Germond $\mathrm{J}-\mathrm{F}$, et al. The advantage of FLASH radiotherapy confirmed in mini-pig and cat-cancer patients. Clin Cancer Res Off J Am Assoc Cancer Res. (2019) 25:35-42. doi: 10.1158/1078-0432.CCR-17-3375

13. Beyreuther E, Brand M, Hans S, Hideghéty K, Karsch L, Leßmann E, et al. Feasibility of proton FLASH effect tested by zebrafish embryo irradiation. Radiother Oncol. (2019) 139:46-50. doi: 10.1016/j.radonc.2019. 06.024

14. Fouillade C, Curras-Alonso S, Giuranno L, Quelennec E, Heinrich S, BonnetBoissinot S, et al. FLASH irradiation spares lung progenitor cells and limits the incidence of radio-induced senescence. Clin Cancer Res. (2020) 26:1497506. doi: 10.1158/1078-0432.CCR-19-1440

15. Montay-Gruel P, Petersson K, Jaccard M, Boivin G, Germond J-F, Petit B, et al. Irradiation in a flash: unique sparing of memory in mice after whole brain irradiation with dose rates above 100 Gy/s. Radiother Oncol. (2017) 124:365-9. doi: 10.1016/j.radonc.2017.05.003

16. Montay-Gruel P, Bouchet A, Jaccard M, Patin D, Serduc R, Aim W, et al. $\mathrm{X}$-rays can trigger the FLASH effect: ultra-high dose-rate synchrotron light source prevents normal brain injury after whole brain irradiation in mice. Radiother Oncol J Eur Soc Ther Radiol Oncol. (2018) 129:582-8. doi: 10.1016/j.radonc.2018.08.016

17. Smyth LML, Donoghue JF, Ventura JA, Livingstone J, Bailey T, Day LRJ, et al. Comparative toxicity of synchrotron and conventional radiation therapy based on total and partial body irradiation in a murine model. Sci Rep. (2018) 8:12044. doi: 10.1038/s41598-018-30543-1

18. Patriarca A, Fouillade C, Auger M, Martin F, Pouzoulet F, Nauraye $\mathrm{C}$, et al. Experimental set-up for FLASH proton irradiation of small animals using a clinical system. Int J Radiat Oncol. (2018) 102:619-26. doi: 10.1016/j.ijrobp.2018.06.403

19. Diffenderfer ES, Verginadis II, Kim MM, Shoniyozov K, Velalopoulou A, Goia D, et al. Design, implementation, and in vivo validation of a novel proton FLASH radiation therapy system. Int J Radiat Oncol. (2020) 106:4408. doi: 10.1016/j.ijrobp.2019.10.049

20. Das IJ, Ding GX, Ahnesjö A. Small fields: nonequilibrium radiation dosimetry: small fields: nonequilibrium radiation dosimetry. Med Phys. (2007) 35:206-15. doi: 10.1118/1.2815356

21. Parwaie W, Refahi S, Ardekani MA, Farhood B. Different dosimeters/detectors used in small-field dosimetry: pros and cons. $J$ Med Signals Sens. (2018) 8:195-203. doi: 10.4103/jmss.JMSS_3_18

22. Grotzer MA, Schültke E, Bräuer-Krisch E, Laissue JA. Microbeam radiation therapy: Clinical perspectives. Phys Med. (2015) 31:564-7. doi: 10.1016/j.ejmp.2015.02.011

23. Bräuer-Krisch E, Adam J-F, Alagoz E, Bartzsch S, Crosbie J, DeWagter $\mathrm{C}$, et al. Medical physics aspects of the synchrotron radiation therapies: microbeam radiation therapy (MRT) and synchrotron stereotactic radiotherapy (SSRT). Phys Med. (2015) 31:568-83. doi: 10.1016/j.ejmp.2015.04.016

24. Draeger E, Sawant A, Johnstone C, Koger B, Becker S, Vujaskovic $Z$, et al. A dose of reality: how 20 years of incomplete physics and dosimetry reporting in radiobiology studies may have contributed to the reproducibility crisis. Int J Radiat Oncol Biol Phys. (2020) 106:243-52. doi: 10.1016/j.ijrobp.2019.06.2545

25. Pedersen KH, Kunugi KA, Hammer CG, Culberson WS, DeWerd LA. Radiation biology irradiator dose verification survey. Radiat Res. (2016) 185:163-8. doi: 10.1667/RR14155.1

26. Desrosiers M, DeWerd L, Deye J, Lindsay P, Murphy MK, Mitch M, et al. The importance of dosimetry standardization in radiobiology. J Res Natl Inst Stand Technol. (2013) 118:403. doi: 10.6028/jres.118.021

27. Wilson JD, Hammond EM, Higgins GS, Petersson K. Ultra-high dose rate (FLASH) radiotherapy: silver bullet or fool's gold? Front Oncol. (2020) 9:1563. doi: 10.3389/fonc.2020.00210

28. Bourhis J, Montay-Gruel P, Gonçalves Jorge P, Bailat C, Petit B, Ollivier J, et al. Clinical translation of FLASH radiotherapy: why and how? Radiother Oncol J Eur Soc Ther Radiol Oncol. (2019) 139:11-7. doi: 10.1016/j.radonc.2019.04.008

29. Favaudon V, Lentz J-M, Heinrich S, Patriarca A, de Marzi L, Fouillade C, et al. Time-resolved dosimetry of pulsed electron beams in very high doserate, FLASH irradiation for radiotherapy preclinical studies. Nucl Instrum Methods Phys Res Sect Accel Spectrometers Detect Assoc Equip. (2019) 944:162537. doi: 10.1016/j.nima.2019.162537

30. Schüler E, Trovati S, King G, Lartey F, Rafat M, Villegas M, et al. Experimental platform for ultra-high dose rate FLASH irradiation of small animals using a clinical linear accelerator. Int J Radiat Oncol Biol Phys. (2017) 97:195-203. doi: 10.1016/j.ijrobp.2016.09.018

31. Kokurewicz K, Brunetti E, Welsh GH, Wiggins SM, Boyd M, Sorensen A, et al. Focused very high-energy electron beams as a novel radiotherapy modality for producing high-dose volumetric elements. Sci Rep. (2019) 9:10837. doi: 10.1038/s41598-019-46630-w

32. Bazalova-Carter M, Qu B, Palma B, Hårdemark B, Hynning E, Jensen C, et al. Treatment planning for radiotherapy with very high-energy electron beams and comparison of VHEE and VMAT plans. Med Phys. (2015) 42:2615-25. doi: $10.1118 / 1.4918923$

33. Eling L, Bouchet A, Nemoz C, Djonov V, Balosso J, Laissue J, et al. Ultra high dose rate synchrotron microbeam radiation therapy. Preclinical evidence in view of a clinical transfer. Radiother Oncol. (2019) 139:56-61. doi: 10.1016/j.radonc.2019.06.030

34. Marlen P van, Dahele M, Folkerts M, Abel E, Slotman BJ, Verbakel WFAR. Bringing FLASH to the clinic: treatment planning considerations for ultrahigh dose-rate proton beams. Int J Radiat Oncol Biol Phys. (2020) 106:621-9. doi: 10.1016/j.ijrobp.2019.11.011

35. Rahman M, Bruza P, Langen KM, Gladstone DJ, Cao X, Pogue BW, et al. Characterization of a new scintillation imaging system for proton pencil beam dose rate measurements. Phys Med Biol. (2020). doi: 10.1088/1361-6560/ab9452. [Epub ahead of print].

36. van de Water S, Safai S, Schippers JM, Weber DC, Lomax AJ. Towards FLASH proton therapy: the impact of treatment planning and machine characteristics on achievable dose rates. Acta Oncol Stockh Swed. (2019) 58:1463-9. doi: 10.1080/0284186X.2019.1627416

37. Jorge PG, Jaccard M, Petersson K, Gondré M, Durán MT, Desorgher L, et al. Dosimetric and preparation procedures for irradiating biological models with pulsed electron beam at ultra-high dose-rate. Radiother Oncol. (2019) 139:34-9. doi: 10.1016/j.radonc.2019.05.004

38. Pratx G, Kapp DS. A computational model of radiolytic oxygen depletion during FLASH irradiation and its effect on the oxygen enhancement ratio. Phys Med Biol. (2019) 64:185005. doi: 10.1088/1361-6560/ab3769

39. Vozenin M-C, Hendry JH, Limoli CL. Biological benefits of ultra-high dose rate FLASH radiotherapy: sleeping beauty awoken. Clin Oncol. (2019) 31:407-15. doi: 10.1016/j.clon.2019.04.001

40. Zhou G. Mechanisms underlying FLASH radiotherapy, a novel way to enlarge the differential responses to ionizing radiation between normal and tumor tissues. Radiat Med Prot. (2020) 1:35-40. doi: 10.1016/j.radmp.2020.02.002

41. Zhou S, Zheng D, Fan Q, Yan Y, Wang S, Lei Y, et al. Minimum dose rate estimation for pulsed FLASH radiotherapy: a dimensional analysis. Med Phys. (2020) 47:3243-9. doi: 10.1002/mp.14181

42. Petersson K, Adrian G, Butterworth K, McMahon SJ. A quantitative analysis of the role of oxygen tension in FLASH radiotherapy. Int J Radiat Oncol. (2020) 107:539-47. doi: 10.1016/j.ijrobp.2020.02.634

43. Weiss H, Epp ER, Heslin JM, Ling CC, Santomasso A. Oxygen depletion in cells irradiated at ultra-high dose-rates and at conventional doserates. Int J Radiat Biol Relat Stud Phys Chem Med. (1974) 26:17-29. doi: 10.1080/09553007414550901

44. Wuerfel JU. Dose measurements in small fields. Med Phys Int. (2013) 1:10. Available online at: http://mpijournal.org/pdf/2013-01/mpi-2013-01-p081. pdf

45. Casar B, Gershkevitsh E, Mendez I, Jurković S, Huq MS. A novel method for the determination of field output factors and output correction factors for small static fields for six diodes and a microdiamond detector in megavoltage photon beams. Med Phys. (2019) 46:944-63. doi: 10.1002/mp.13318

46. Palmans H, Andreo P, Huq MS, Seuntjens J, Christaki KE, Meghzifene A. Dosimetry of small static fields used in external photon beam radiotherapy: 
summary of TRS-483, the IAEA-AAPM international code of practice for reference and relative dose determination. Med Phys. (2018) 45:e1123-45. doi: $10.1002 / \mathrm{mp} .13208$

47. Boag JW. The recombination correction for an ionisation chamber exposed to pulsed radiation in a 'beam' technique I. Theory Phys Med Biol. (1982) 27:201-11. doi: 10.1088/0031-9155/27/2/001

48. Petersson K, Jaccard M, Germond J-F, Buchillier T, Bochud F, Bourhis J, et al. High dose-per-pulse electron beam dosimetry - A model to correct for the ion recombination in the advanced markus ionization chamber. Med Phys. (2017) 44:1157-67. doi: 10.1002/mp.12111

49. Brualla-González L, Gómez F, Pombar M, Pardo-Montero J. Dose rate dependence of the PTW 60019 microDiamond detector in high dose-per-pulse pulsed beams. Phys Med Biol. (2016) 61:N11-9. doi: 10.1088/0031-9155/61/1/N11

50. Saini AS, Zhu TC. Dose rate and SDD dependence of commercially available diode detectors. Med Phys. (2004) 31:914-24. doi: 10.1118/1.1650563

51. Fowler JF. Radiation-induced conductivity in the solid state, and some applications. Phys Med Biol. (1959) 3:395-410. doi: 10.1088/0031-9155/3/4/307

52. Ade N, Nam TL, Derry TE, Mhlanga SH. The dose rate dependence of synthetic diamond detectors in the relative dosimetry of highenergy electron therapy beams. Radiat Phys Chem. (2014) 98:155-62. doi: 10.1016/j.radphyschem.2014.02.003

53. Fidanzio A, Azario L, Miceli R, Russo A, Piermattei A. PTW-diamond detector: dose rate and particle type dependence. Med Phys. (2000) 27:258993. doi: 10.1118/1.1318218

54. Jursinic PA. Dependence of diode sensitivity on the pulse rate of delivered radiation. Med Phys. (2013) 40:021720. doi: 10.1118/1.4788763

55. Neira S, Brualla-Gónzalez L, Prieto-Pena J, Gómez F, Pardo-Montero J. A kinetic model of diode detector response to pulsed radiation beams. Phys Med Biol. (2019) 64:205007. doi: 10.1088/1361-6560/ab4460

56. Shi J, Simon WE, Zhu TC. Modeling the instantaneous dose rate dependence of radiation diode detectors. Med Phys. (2003) 30:2509-19. doi: 10.1118/1.1602171

57. Müller O, Stötzel J, Lützenkirchen-Hecht D, Frahm R. Gridded ionization chambers for time resolved X-Ray absorption spectroscopy. J Phys Conf Ser. (2013) 425:092010. doi: 10.1088/1742-6596/425/9/092010

58. Schroder DK. Carrier lifetimes in silicon. IEEE Trans Electron Devices. (1997) 44:160-70. doi: 10.1109/16.554806

59. Kozlov SF, Stuck R, Hage-Ali M, Siffert P. Preparation and characteristics of natural diamond nuclear radiation detectors. IEEE Trans Nucl Sci. (1975) 22:160-70. doi: 10.1109/TNS.1975.4327634

60. Isberg J, Hammersberg J, Johansson E, Wikström T, Twitchen DJ, Whitehead AJ, et al. High carrier mobility in single-crystal plasma-deposited diamond. Science. (2002) 297:1670-2. doi: 10.1126/science.1074374

61. Hendry JH, Moore JV, Hodgson BW, Keene JP. The constant low oxygen concentration in all the target cells for mouse tail radionecrosis. Radiat Res. (1982) 92:172-81. doi: 10.2307/3575852

62. Hu J, Rovey JL. Faraday cup with nanosecond response and adjustable impedance for fast electron beam characterization. Rev Sci Instrum. (2011) 82:073504. doi: 10.1063/1.3610649

63. Richter C, Karsch L, Dammene Y, Kraft SD, Metzkes J, Schramm U, et al. A dosimetric system for quantitative cell irradiation experiments with laser-accelerated protons. Phys Med Biol. (2011) 56:1529-1543. doi: 10.1088/0031-9155/56/6/002

64. Prokupek J, Kaufman J, Margarone D, Krus M, Velyhan A, Krása J, et al. Development and first experimental tests of Faraday cup array. Rev Sci Instrum. (2014) 85:013302. doi: 10.1063/1.4859496

65. Lerch MLF, Petasecca M, Cullen A, Hamad A, Requardt H, Bräuer-Krisch E, et al. Dosimetry of intensive synchrotron microbeams. Radiat Meas. (2011) 46:1560-5. doi: 10.1016/j.radmeas.2011.08.009

66. O'Leary M, Boscolo D, Breslin N, Brown JMC, Dolbnya IP, Emerson C, et al. Observation of dose-rate dependence in a Fricke dosimeter irradiated at low dose rates with monoenergetic X-rays. Sci Rep. (2018) 8:1-9. doi: 10.1038/s41598-018-21813-z

67. Anton M. Uncertainties in alanine/ESR dosimetry at the physikalischtechnische bundesanstalt. Phys Med Biol. (2006) 51:5419-40. doi: $10.1088 / 0031-9155 / 51 / 21 / 003$
68. Kudoh H, Celina M, Kaye RJ, Gillen KT, Clough RL. Response of alanine dosimeters at very high dose rate. Appl Radiat Isot. (1997) 48:497-9. doi: 10.1016/S0969-8043(96)00281-3

69. Soliman YS, Pellicioli P, Beshir WB, Abdel-Fattah AA, Fahim RA, Krisch $\mathrm{M}$, et al. A comparative dosimetry study of an alanine dosimeter with a PTW PinPoint chamber at ultra-high dose rates of synchrotron radiation. Phys Med Eur J Med Phys. (2020) 71:161-7. doi: 10.1016/j.ejmp.2020. 03.007

70. Karsch L, Beyreuther E, Burris-Mog T, Kraft S, Richter C, Zeil K, et al. Dose rate dependence for different dosimeters and detectors: TLD, OSL, EBT films, and diamond detectors. Med Phys. (2012) 39:2447-55. doi: $10.1118 / 1.3700400$

71. Jaccard M, Petersson K, Buchillier T, Germond J-F, Durán MT, Vozenin $\mathrm{M}-\mathrm{C}$, et al. High dose-per-pulse electron beam dosimetry: usability and doserate independence of EBT3 gafchromic films. Med Phys. (2017) 44:725-35. doi: $10.1002 / \mathrm{mp} .12066$

72. McLaughlin WL, Al-Sheikhly M, Lewis DF, Kovács A, Wojnárovits L. Radiochromic solid-state polymerization reaction. In: Clough RL, Shalaby SW, editors. Irradiation of Polymers. American Che mical Society. (1996). p. 152-66.

73. Casolaro P, Campajola L, Breglio G, Buontempo S, Consales M, Cusano A, et al. Real-time dosimetry with radiochromic films. Sci Rep. (2019) 9:5307. doi: 10.1038/s41598-019-41705-0

74. Rink A, Vitkin IA, Jaffray DA. Suitability of radiochromic medium for realtime optical measurements of ionizing radiation dose. Med Phys. (2005) 32:1140-55. doi: 10.1118/1.1877832

75. Baldock C, De Deene Y, Doran S, Ibbott G, Jirasek A, Lepage M, et al. Polymer gel dosimetry. Phys Med Biol. (2010) 55:R1-63. doi: 10.1088/0031-9155/55/5/R01

76. Deene YD. Essential characteristics of polymer gel dosimeters. J Phys Conf Ser. (2004) 3:34-57. doi: 10.1088/1742-6596/3/1/006

77. Khan M, Heilemann G, Kuess P, Georg D, Berg A. The impact of the oxygen scavenger on the dose-rate dependence and dose sensitivity of MAGIC type polymer gels. Phys Med Biol. (2018) 63:06NT01. doi: 10.1088/1361-6560/aab00b

78. McKeever SWS. Optically stimulated luminescence: a brief overview. Radiat Meas. (2011) 46:1336-41. doi: 10.1016/j.radmeas.2011.02.016

79. Inada T, Nishio H, Amino S, Abe K, Saito K. High dose-rate dependence of early skin reaction in mouse. Int J Radiat Biol Relat Stud Phys Chem Med. (1980) 38:139-45. doi: 10.1080/09553008014551031

80. Karzmark CJ, White J, Fowler JF. Lithium fluoride thermoluminescence dosimetry. Phys Med Biol. (1964) 9:273-86. doi: 10.1088/0031-9155/9/3/302

81. Tochilin E, Goldstein N. Dose rate and spectral measurements from pulsed x-ray generators. Health Phys. (1966) 12:1705-14. doi: 10.1097/00004032-196612000-00007

82. Ptaszkiewicz M, Braurer-Kirsch E, Klosowski M, Czopyk L, Olko P. TLD dosimetry for microbeam radiation therapy at the European synchrotron radiation facility. Radiat Meas. (2008) 43:990-3. doi: 10.1016/j.radmeas.2007.12.050

83. Ahmed MF, Shrestha N, Ahmad S, Schnell E, Akselrod MS, Yukihara EG. Demonstration of 2D dosimetry using $\mathrm{Al} 2 \mathrm{O} 3$ optically stimulated luminescence films for therapeutic megavoltage $\mathrm{x}$-ray and ion beams. Radiat Meas. (2017) 106:315-20. doi: 10.1016/j.radmeas.2017.04.010

84. Wouter C, Dirk V, Paul L, Tom D. A reusable OSL-film for 2D radiotherapy dosimetry. Phys Med Biol. (2017) 62:8441-54. doi: 10.1088/1361-6560/aa8de6

85. Akselrod MS, Sykora GJ. Fluorescent nuclear track detector technology - A new way to do passive solid state dosimetry. Radiat Meas. (2011) 46:1671-9. doi: 10.1016/j.radmeas.2011.06.018

86. Bartz JA, Sykora GJ, Bräuer-Krisch E, Akselrod MS. Imaging and dosimetry of synchrotron microbeam with aluminum oxide fluorescent detectors. Radiat Meas. (2011) 46:1936-9. doi: 10.1016/j.radmeas.2011.04.003

87. Birks JB. The Theory and Practice of Scintillation Counting. Oxford: Pergamon Press (1964). p. 671.

88. Beddar S, Beaulmieu L. Scintillation Dosimetry. Boca Raton, FL: CRC Press (2016). p. 424.

89. Nikl M. Scintillation detectors for x-rays. Meas Sci Technol. (2006) 17:R3754. doi: 10.1088/0957-0233/17/4/R01 
90. Horrocks DL. Applications of Liquid Scintillation Counting. New York, NY; London: Academic Press (1974). p. 357.

91. Peng C-T, Horrocks DL, Alpen EL. Liquid Scintillation Counting: Recent Applications and Development. Cambridge, MA: Academic Press (1980). p. 456.

92. Benmakhlouf $\mathrm{H}$, Andreo P. Spectral distribution of particle fluence in small field detectors and its implication on small field dosimetry. Med Phys. (2017) 44:713-24. doi: 10.1002/mp.12042

93. Archer J, Li E, Davis J, Cameron M, Rosenfeld A, Lerch M. High spatial resolution scintillator dosimetry of synchrotron microbeams. Sci Rep. (2019) 9:1-7. doi: 10.1038/s41598-019-43349-6

94. Beddar S, Archambault L, Sahoo N, Poenisch F, Chen GT, Gillin MT, et al. Exploration of the potential of liquid scintillators for real-time 3D dosimetry of intensity modulated proton beams. Med Phys. (2009) 36:173643. doi: $10.1118 / 1.3117583$

95. Pönisch F, Archambault L, Briere TM, Sahoo N, Mohan R, Beddar $\mathrm{S}$, et al. Liquid scintillator for 2D dosimetry for high-energy photon beams: Liquid scintillator for 2D dosimetry. Med Phys. (2009) 36:1478-85. doi: 10.1118/1.3106390

96. Ashraf MR, Bruza P, Pogue BW, Nelson N, Williams BB, Jarvis LA, et al. Optical imaging provides rapid verification of static small beams, radiosurgery, and VMAT plans with millimeter resolution. Med Phys. (2019) 46:5227-37. doi: 10.1002/mp.13797

97. Vigdor SE, Klyachko AV, Solberg KA, Pankuch M. A gas scintillator detector for 2D dose profile monitoring in pencil beam scanning and pulsed beam proton radiotherapy treatments. Phys Med Biol. (2017) 62:4946-69. doi: 10.1088/1361-6560/aa6ce2

98. Darne CD, Alsanea F, Robertson DG, Hojo Y, Sahoo N, Beddar S. 3D scintillator detector system for proton scanning beam therapy. Int J Radiat Oncol Biol Phys. (2019) 105:S89-90. doi: 10.1016/j.ijrobp.2019.06.563

99. Darne CD, Alsanea F, Robertson DG, Sahoo N, Beddar S. Performance characterization of a 3D liquid scintillation detector for discrete spot scanning proton beam systems. Phys Med Biol. (2017) 62:5652-67. doi: 10.1088/1361-6560/aa780b

100. Goulet M, Rilling M, Gingras L, Beddar S, Beaulieu L, Archambault L. Novel, full 3D scintillation dosimetry using a static plenoptic camera. Med Phys. (2014) 41:082101. doi: 10.1118/1.4884036

101. Kirov AS, Piao JZ, Mathur NK, Miller TR, Devic S, Trichter S, et al. The three-dimensional scintillation dosimetry method: test for a 106Ru eye plaque applicator. Phys Med Biol. (2005) 50:3063-81. doi: 10.1088/0031-9155/50/13/007

102. Beddar S. Real-time volumetric scintillation dosimetry. J Phys Conf Ser. (2015) 573:012005. doi: 10.1088/1742-6596/573/1/012005

103. Bruza P, Andreozzi JM, Gladstone DJ, Jarvis LA, Rottmann J, Pogue BW. Online combination of EPID cherenkov imaging for 3-D dosimetry in a liquid phantom. IEEE Trans Med Imaging. (2017) 36:2099-103. doi: 10.1109/TMI.2017.2717800

104. Glaser AK, Zhang R, Gladstone DJ, Pogue BW. Optical dosimetry of radiotherapy beams using Cherenkov radiation: the relationship between light emission and dose. Phys Med Biol. (2014) 59:3789-811. doi: 10.1088/0031-9155/59/14/3789

105. Glaser AK, Davis SC, McClatchy DM, Zhang R, Pogue BW, Gladstone DJ. Projection imaging of photon beams by the Cerenkov effect: projection imaging of photon beams by the Cerenkov effect. Med Phys. (2012) 40:012101. doi: 10.1118/1.4770286

106. Brunner SE, Schaart DR. BGO as a hybrid scintillator / Cherenkov radiator for cost-effective time-of-flight PET. Phys Med Biol. (2017) 62:4421-39. doi: 10.1088/1361-6560/aa6a49

107. Brunner SE, Gruber L, Marton J, Suzuki K, Hirtl A. Studies on the Cherenkov effect for improved time resolution of TOF-PET. IEEE Trans Nucl Sci. (2014) 61:443-7. doi: 10.1109/TNS.2013.2281667

108. Cates JW, Levin CS. Evaluation of a clinical TOF-PET detector design that achieves $\leqslant 100$ ps coincidence time resolution. Phys Med Biol. (2018) 63:115011. doi: 10.1088/1361-6560/aac504

109. Grigoryants VM, Lozovoy VV, Chernousov YuD, Shebolaev IV, Arutyunov VA, Anisimov OA, et al. Pulse radiolysis system with picosecond time resolution referred to Cherenkov radiation. Int J Radiat Appl Instrum
Part C Radiat Phys Chem. (1989) 34:349-52. doi: 10.1016/1359-0197(89) 90243-9

110. Yang J, Kondoh T, Yoshida A, Yoshida Y. Double-decker femtosecond electron beam accelerator for pulse radiolysis. Rev Sci Instrum. (2006) 77:043302. doi: 10.1063/1.2195090

111. Glaser AK, Andreozzi JM, Davis SC, Zhang R, Pogue BW, Fox CJ, et al. Video-rate optical dosimetry and dynamic visualization of IMRT and VMAT treatment plans in water using Cherenkov radiation. Med Phys. (2014) 41:062102. doi: 10.1118/1.4875704

112. Jarvis LA, Zhang R, Gladstone DJ, Jiang S, Hitchcock W, Friedman OD, et al. Cherenkov video imaging allows for the first visualization of radiation therapy in real time. Int J Radiat Oncol Biol Phys. (2014) 89:615-22. doi: 10.1016/j.ijrobp.2014.01.046

113. Adrian G, Konradsson E, Lempart M, Bäck S, Ceberg C, Petersson K. The FLASH effect depends on oxygen concentration. Br J Radiol. (2020) 93:20190702. doi: 10.1259/bjr.20190702

114. Richardson RB. Age-dependent changes in oxygen tension, radiation dose and sensitivity within normal and diseased coronary arteries-Part C: oxygen effect and its implications on high- and low-LET dose. Int J Radiat Biol. (2008) 84:858-65. doi: 10.1080/09553000802389686

115. Boscolo D, Krämer M, Fuss MC, Durante M, Scifoni E. Impact of target oxygenation on the chemical track evolution of ion and electron radiation. Int J Mol Sci. (2020) 21:424. doi: 10.3390/ijms21020424

116. Hockel M, Vaupel P. Tumor hypoxia: definitions and current clinical, biologic, and molecular aspects. JNCI J Natl Cancer Inst. (2001) 93:266-76. doi: 10.1093/jnci/93.4.266

117. Horsman MR, Mortensen LS, Petersen JB, Busk M, Overgaard J. Imaging hypoxia to improve radiotherapy outcome. Nat Rev Clin Oncol. (2012) 9:674-87. doi: 10.1038/nrclinonc.2012.171

118. Vaupel P, Kelleher DK, Houmlckel M. Oxygenation status of malignant tumors: pathogenesis of hypoxia and significance for tumor therapy. Semin Oncol. (2001) 28:29-35. doi: 10.1016/S0093-7754(01)90210-6

119. Sørensen M, Horsman MR, Cumming P, Munk OL, Keiding S. Effect of intratumoral heterogeneity in oxygenation status on FMISO PET, autoradiography, and electrode Po2 measurements in murine tumors. Int $J$ Radiat Oncol. (2005) 62:854-61. doi: 10.1016/j.ijrobp.2005.02.044

120. Nordsmark M, Bentzen SM, Overgaard J. Measurement of human tumour oxygenation status by a polarographic needle electrode: an analysis of inter- and intratumour heterogeneity. Acta Oncol. (1994) 33:383-9. doi: 10.3109/028418694090 98433

121. Nozue M, Lee I, Yuan F, Teicher BA, Brizel DM, Dewhirst MW, et al. Interlaboratory variation in oxygen tension measurement by Eppendorf "histograph" and comparison with hypoxic marker. J Surg Oncol. (1997) 66:30-8. doi: 10.1002/(sici)1096-9098(199709)66:1<30::aid-jso7>3.0.co;2-o

122. Collingridge DR, Young WK, Vojnovic B, Wardman P, Lynch EM, Hill SA, et al. Measurement of tumor oxygenation: a comparison between polarographic needle electrodes and a time-resolved luminescence-based optical sensor. Radiat Res. (1997) 147:329. doi: 10.2307/3579340

123. Zhao D, Jiang L, Hahn EW, Mason RP. Tumor physiologic response to combretastatin A4 phosphate assessed by MRI. Int J Radiat Oncol. (2005) 62:872-80. doi: 10.1016/j.ijrobp.2005.03.009

124. Swartz HM, Williams BB, Zaki BI, Hartford AC, Jarvis LA, Chen EY, et al. Clinical EPR: unique opportunities and some challenges. Acad Radiol. (2014) 21:197-206. doi: 10.1016/j.acra.2013.10.011

125. Khan N, Williams BB, Hou H, Li H, Swartz HM. Repetitive tissue pO2 measurements by electron paramagnetic resonance oximetry: current status and future potential for experimental and clinical studies. Antioxid Redox Signal. (2007) 9:1169-82. doi: 10.1089/ars.2007.1635

126. Pogue BW, Feng J, LaRochelle EP, BruŽa P, Lin H, Zhang R, et al. Maps of in vivo oxygen pressure with submillimetre resolution and nanomolar sensitivity enabled by Cherenkov-excited luminescence scanned imaging. Nat Biomed Eng. (2018) 2:254-64. doi: 10.1038/s41551-018-0220-3

127. Cao X, Rao Allu S, Jiang S, Jia M, Gunn JR, Yao C, et al. Tissue pO2 distributions in xenograft tumors dynamically imaged by Cherenkov-excited phosphorescence during fractionated radiation therapy. Nat Commun. (2020) 11:573. doi: 10.1038/s41467-020-14415-9 
128. Zhang R, D'souza AV, Gunn JR, Esipova TV, Vinogradov SA, Glaser AK, et al. Cherenkov-excited luminescence scanned imaging. Opt Lett. (2015) 40:827. doi: 10.1364/OL.40.000827

129. BruŽa P, Lin H, Vinogradov SA, Jarvis LA, Gladstone DJ, Pogue BW. Light sheet luminescence imaging with Cherenkov excitation in thick scattering media. Opt Lett. (2016) 41:2986. doi: 10.1364/OL.41.0 02986

130. Bertolet A, Cortés-Giraldo MA, Carabe-Fernandez A. On the concepts of dose-mean lineal energy, unrestricted and restricted doseaveraged LET in proton therapy. Phys Med Biol. (2020) 65:075011. doi: 10.1088/1361-6560/ab730a

131. Cortés-Giraldo MA, Carabe A. A critical study of different Monte Carlo scoring methods of dose average linear-energy-transfer maps calculated in voxelized geometries irradiated with clinical proton beams. Phys Med Biol. (2015) 60:2645-69. doi: 10.1088/0031-9155/60/ $7 / 2645$

132. Sawakuchi GO, Ferreira FA, McFadden CH, Hallacy TM, Granville DA, Sahoo N, et al. Nanoscale measurements of proton tracks using fluorescent nuclear track detectors: nanoscale measurements of proton tracks. Med Phys. (2016) 43:2485-90. doi: 10.1118/1.4947128

133. Granville DA, Sahoo N, Sawakuchi GO. Simultaneous measurements of absorbed dose and linear energy transfer in therapeutic proton beams. Phys Med Biol. (2016) 61:1765-79. doi: 10.1088/0031-9155/61/ 4/1765

134. Sawakuchi GO, Sahoo N, Gasparian PBR, Rodriguez MG, Archambault L, Titt U, et al. Determination of average LET of therapeutic proton beams using $\mathrm{Al}_{2} \mathrm{O}_{3}$ : $\mathrm{C}$ optically stimulated luminescence (OSL) detectors. Phys Med Biol. (2010) 55:4963-76. doi: 10.1088/0031-9155/55/ $17 / 006$

135. Alsanea F, Therriault-Proulx F, Sawakuchi G, Beddar S. A real-time method to simultaneously measure linear energy transfer and dose for proton therapy using organic scintillators. Med Phys. (2018) 45:1782-9. doi: $10.1002 / \mathrm{mp} .12815$

136. Terasawa K, Borak TB, Doke T, Fuse T, Hara K, Kikuchi J, et al. The response of the silicon-based linear energy transfer spectrometer (RRMDIII) to protons from 1 to $70 \mathrm{MeV}$. Jpn J Appl Phys. (2005) 44:7608-13. doi: 10.1143/JJAP.44.7608
137. Kry SF, Alvarez P, Cygler JE, DeWerd LA, Howell RM, Meeks S, et al. AAPM TG 191: clinical use of luminescent dosimeters: TLDs and OSLDs. Med Phys. (2020) 47:e19-51. doi: 10.1002/mp.13839

138. Fukumura A, Hoshino K, Takeshita M, Kanai T, Minohara S, Sudou M. Silicon Diodes as Detectors in Relative Dosimetry of Heavy Ions. (1994). p. 8. Available online at: https://inis.iaea.org/search/search.aspx?orig_q=RN: 27036816

139. Bourhis J, Sozzi WJ, Jorge PG, Gaide O, Bailat C, Duclos F, et al. Treatment of a first patient with FLASH-radiotherapy. Radiother Oncol. (2019) 139:18-22. doi: 10.1016/j.radonc.2019.06.019

140. Venkatesulu BP, Sharma A, Pollard-Larkin JM, Sadagopan R, Symons J, Neri $\mathrm{S}$, et al. Ultra high dose rate $(35 \mathrm{~Gy} / \mathrm{sec})$ radiation does not spare the normal tissue in cardiac and splenic models of lymphopenia and gastrointestinal syndrome. Sci Rep. (2019) 9:1-9. doi: 10.1038/s41598-019-53562-y

141. Pappas EP, Zoros E, Zourari K, Hourdakis CI, Papagiannis P, Karaiskos P, et al. PO-0774: Investigation of dose-rate dependence at an extensive range for PRESAGE radiochromic dosimeter. Radiother Oncol. (2017) 123:S410. doi: 10.1016/S0167-8140(17)31211-2

142. Sellakumar P, Samuel EJJ, Kumar DS. Dose-rate dependence of PAGAT polymer gel dosimeter evaluated using X-ray CT scanner. J Phys Conf Ser. (2010) 250:012074. doi: 10.1088/1742-6596/250/1/012074

143. Ashraf MR, Rahman M, Zhang R, Williams BB, Gladstone DJ, Pogue BW, et al. Dosimetry for FLASH radiotherapy: a review of tools and the role of radioluminescence and cherenkov emission. ArXiv200603755 (Preprint) Phys. (2020).

Conflict of Interest: The authors declare that the research was conducted in the absence of any commercial or financial relationships that could be construed as a potential conflict of interest.

Copyright (c) 2020 Ashraf, Rahman, Zhang, Williams, Gladstone, Pogue and Bruza. This is an open-access article distributed under the terms of the Creative Commons Attribution License (CC BY). The use, distribution or reproduction in other forums is permitted, provided the original author(s) and the copyright owner(s) are credited and that the original publication in this journal is cited, in accordance with accepted academic practice. No use, distribution or reproduction is permitted which does not comply with these terms. 